\title{
Structure of Essential Spectrum and Discrete Spectra of the Energy Operator of Five-Electron Systems in the Hubbard Model. Third and Fourth Doublet States
}

\author{
S. M. Tashpulatov \\ Institute of Nuclear Physics of Academy of Science of Republic of Uzbekistan, Tashkent, Uzbekistan \\ Email: sadullatashpulatov@yandex.ru, toshpul@mail.ru, toshpul@inp.uz
}

How to cite this paper: Tashpulatov, S.M. (2020) Structure of Essential Spectrum and Discrete Spectra of the Energy Operator of Five-Electron Systems in the Hubbard Model. Third and Fourth Doublet States. Journal of Applied Mathematics and Physics, 8, 2886-2918.

https://doi.org/10.4236/jamp.2020.812214

Received: October 21, 2020

Accepted: December 15, 2020

Published: December 18, 2020

Copyright $\odot 2020$ by author(s) and Scientific Research Publishing Inc. This work is licensed under the Creative Commons Attribution International License (CC BY 4.0).

http://creativecommons.org/licenses/by/4.0/

\begin{abstract}
We consider a five-electron system in the Hubbard model with a coupling between nearest-neighbors. The structure of essential spectrum and discrete spectrum of the systems in the third and fourth doublet states in a $v$-dimensional lattice is investigated. We prove that the essential spectrum of the system in a third doublet state consists is the union of at most four segments, and discrete spectrum of the system is empty. We show that the essential spectrum of the system in a fourth doublet state consists of the union of at most seven segments, and discrete spectrum of the system consists of no more than one point.
\end{abstract}

\section{Keywords}

Essential Spectrum, Discrete Spectrum, Five-Electron System, Bound State, Anti-Bound State, Hubbard Model, Doublet State, Sextet State, Quartet State

\section{Introduction}

In the early 1970s, three papers [1] [2] [3], where a simple model of a metal was proposed that has become a fundamental model in the theory of strongly correlated electron systems, appeared almost simultaneously and independently. In that model, a single nondegenerate electron band with a local Coulomb interaction is considered. The model Hamiltonian contains only two parameters: the matrix element $t$ of electron hopping from a lattice site to a neighboring site and the parameter $U$ of the on-site Coulomb repulsion of two electrons. In the secondary quantization representation, the Hamiltonian can be written as 


$$
H=t \sum_{m, \gamma} a_{m, \gamma}^{+} a_{m, \gamma}+U \sum_{m} a_{m, \uparrow}^{+} a_{m, \uparrow} a_{m, \downarrow}^{+} a_{m, \downarrow}
$$

where $a_{m, \gamma}^{+}$and $a_{m, \gamma}$ denote Fermi operators of creation and annihilation of an electron with spin $\gamma$ on a site $m$ and the summation over $\tau$ means summation over the nearest neighbors on the lattice.

The model proposed in [1] [2] [3] was called the Hubbard model after John Hubbard, who made a fundamental contribution to studying the statistical mechanics of that system, although the local form of Coulomb interaction was first introduced for an impurity model in a metal by Anderson [4]. We also recall that the Hubbard model is a particular case of the Shubin-Wonsowsky polaron model [5], which had appeared 30 years before [1] [2] [3]. In the Shubin-Wonsowsky model, along with the on-site Coulomb interaction, the interaction of electrons on neighboring sites is also taken into account. The Hubbard model is an approximation used in solid state physics to describe the transition between conducting and insulating states. It is the simplest model describing particle interaction on a lattice. Its Hamiltonian contains only two terms: the kinetic term corresponding to the tunneling (hopping) of particles between lattice sites and a term corresponding to the on-site interaction. Particles can be fermions, as in Hubbard's original work, and also bosons. The simplicity and sufficiency of Hamiltonian (1) have made the Hubbard model very popular and effective for describing strongly correlated electron systems.

The Hubbard model well describes the behavior of particles in a periodic potential at sufficiently low temperatures such that all particles are in the lower Bloch band and long-range interactions can be neglected. If the interaction between particles on different sites is taken into account, then the model is often called the extended Hubbard model. It was proposed for describing electrons in solids, and it remains especially interesting since then for studying high-temperature superconductivity. Later, the extended Hubbard model also found applications in describing the behavior of ultracold atoms in optical lattices. In considering electrons in solids, the Hubbard model can be considered a sophisticated version of the model of strongly bound electrons, involving only the electron hopping term in the Hamiltonian. In the case of strong interactions, these two models can give essentially different results. The Hubbard model exactly predicts the existence of so-called Mott insulators, where conductance is absent due to strong repulsion between particles. The Hubbard model is based on the approximation of strongly coupled electrons. In the strong coupling approximation, electrons initially occupy orbitals in atoms (lattice sites) and then hop over to other atoms, thus conducting the current. Mathematically, this is represented by the so-called hopping integral. This process can be considered the physical phenomenon underlying the occurrence of electron bands in crystal materials. But the interaction between electrons is not considered in more general band theories. In addition to the hopping integral, which explains the conductance of the material, the Hubbard model contains the so-called on-site repulsion, corresponding to the Coulomb repulsion between electrons. This leads to a competition between the 
hopping integral, which depends on the mutual position of lattice sites, and the on-site repulsion, which is independent of the atom positions. As a result, the Hubbard model explains the metal-insulator transition in oxides of some transition metals. When such a material is heated, the distance between nearest-neighbor sites increases, the hopping integral decreases, and on-site repulsion becomes dominant.

The Hubbard model is currently one of the most extensively studied multielectron models of metals [6] [7] [8] [9] [10]. But little is known about exact results for the spectrum and wave functions of the crystal described by the Hubbard model, and obtaining the corresponding statements is therefore of great interest. The spectrum and wave functions of the system of two electrons in a crystal described by the Hubbard Hamiltonian were studied in [6]. It is known that two-electron systems can be in two states, triplet and singlet [6] [7] [8] [9] [10]. It was proved in [6] that the spectrum of the system Hamiltonian $H^{t}$ in the triplet state is purely continuous and coincides with a segment $[m, M]$, and the operator $H^{s}$ of the system in the singlet state, in addition to the continuous spectrum $[m, M]$, has a unique antibound state for some values of the quasimomentum. For the antibound state, correlated motion of the electrons is realized under which the contribution of binary states is large. Because the system is closed, the energy must remain constant and large. This prevents the electrons from being separated by long distances. Next, an essential point is that bound states (sometimes called scattering-type states) do not form below the continuous spectrum. This can be easily understood because the interaction is repulsive. We note that a converse situation is realized for $U<0$ : below the continuous spectrum, there is a bound state (antibound states are absent) because the electrons are then attracted to one another.

For the first band, the spectrum is independent of the parameter $U$ of the on-site Coulomb interaction of two electrons and corresponds to the energy of two noninteracting electrons, being exactly equal to the triplet band. The second band is determined by Coulomb interaction to a much greater degree: both the amplitudes and the energy of two electrons depend on $U$, and the band itself disappears as $U \rightarrow 0$ and increases without bound as $U \rightarrow \infty$. The second band largely corresponds to a one-particle state, namely, the motion of the doublet, i.e., two-electron bound states.

The spectrum and wave functions of the system of three electrons in a crystal described by the Hubbard Hamiltonian were studied in [11]. In the three-electron systems are exists quartet state, and two type doublet states. In [11] proved the essential spectrum of the system in a quartet state consists of a single segment and the three-electron bound states or three-electron anti-bound states is absent. Furthermore, shown what the essential spectrum of the system in doublet states is the union of at most three segments. In [11] also proved that three-electron bound states exist in doublet states.

The spectrum of the energy operator of system of four electrons in a crystal described by the Hubbard Hamiltonian in the triplet state was studied in [12]. In 
the four-electron systems exist quintet state, and three type triplet states, and two type singlet states. In the work [12] proved that the essential spectrum of the system in a triplet state is the union of at most three segments. There are also proved that the four-electron bound states or a four-electron anti-bound states exists in triplet states. Furthermore, also proved the spectrum of these three triplet states are different. The spectrum of the energy operator of four-electron systems in the Hubbard model in the quintet, and singlet states were studied in [13]. In [13] proved the essential spectrum of the system in a quintet state consists of a single segment and the four-electron bound states or four-electron anti-bound states is absent. In the system exists two type four-electron singlet states, and they are different origins. In the singlet states the essential spectra of four-electron systems consist of the union of no more than three segments. Furthermore, in the system exists no more one four-electron anti-bound states or no more one bound states.

Here, we consider the energy operator of five-electron systems in the Hubbard model and describe the structure of the essential spectra and discrete spectrum of the system for third and fourth doublet states.

The Hamiltonian of the chosen model has the form

$$
H=A \sum_{m, \gamma} a_{m, \gamma}^{+} a_{m, \gamma}+B \sum_{m, \tau, \gamma} a_{m+\tau, \gamma}^{+} a_{m, \gamma}+U \sum_{m} a_{m, \uparrow}^{+} a_{m, \uparrow} a_{m, \downarrow}^{+} a_{m, \downarrow} .
$$

Here, $A$ is the electron energy at a lattice site, $B$ is the transfer integral between neighboring sites (we assume that $B>0$ for convenience), $\tau= \pm e_{j}, j=1,2, \cdots, v$, where $e_{j}$ are unit mutually orthogonal vectors, which means that summation is taken over the nearest neighbors, $U$ is the parameter of the on-site Coulomb interaction of two electrons, $\gamma$ is the spin index, $\gamma=\uparrow$ or $\gamma=\downarrow, \uparrow$ and $\downarrow$ denote the spin values $\frac{1}{2}$ and $-\frac{1}{2}$, and $a_{m, \gamma}^{+}$and $a_{m, \gamma}$ are the respective electron creation and annihilation operators at a site $m \in Z^{v}$.

In the five-electron systems exists sextet state, four type quartet states, and five type doublet states.

The energy of the system depends on its total spin $S$. Along with the Hamiltonian, the $N_{e}$ electron system is characterized by the total spin $S$, $S=S_{\max }, S_{\max }-1, \cdots, S_{\min }, S_{\max }=\frac{N_{e}}{2}, S_{\min }=0, \frac{1}{2}$.

Hamiltonian (2) commutes with all components of the total spin operator $S=\left(S^{+}, S^{-}, S^{z}\right)$, and the structure of eigenfunctions and eigenvalues of the system therefore depends on $S$. The Hamiltonian $H$ acts in the antisymmetric Fo'ck space $\mathcal{H}_{a s}$.

Belove we will give constructions of the Fo'ck space.

Let $\mathcal{H}$ be a Hilbert space and denote by $\mathcal{H}^{n}$ the $n$-fold tensor product $\mathcal{H}^{n}=\mathcal{H} \otimes \mathcal{H} \otimes \cdots \otimes \mathcal{H}$. We set $\mathcal{H}^{0}=C$ and define $\mathcal{F}(\mathcal{H})=\bigoplus_{n=0}^{\infty} \mathcal{H}^{n}$ The $\mathcal{F}(\mathcal{H})$ is called the Fock space over $\mathcal{H}$; it will be separable, if $\mathcal{H}$ is. For example, if $\mathcal{H}=L_{2}(R)$, then an element $\psi \in \mathcal{F}(\mathcal{H})$ is a sequence of functions $\psi=\left\{\psi_{0}, \psi_{1}\left(x_{1}\right), \psi_{2}\left(x_{1}, x_{2}\right), \psi_{3}\left(x_{1}, x_{2}, x_{3}\right), \cdots\right\}$, so that 


$$
\left|\psi_{0}\right|^{2}+\sum_{n=1}^{\infty} \int_{R^{n}}\left|\psi_{n}\left(x_{1}, x_{2}, \cdots, x_{n}\right)\right|^{2} \mathrm{~d} x_{1} \mathrm{~d} x_{2} \cdots \mathrm{d} x_{n}<\infty .
$$

Actually, it is not $\mathcal{F}(\mathcal{H})$ itself, but two of its subspaces which are used most frequently in quantum field theory. These two subspaces are constructed as follows: Let $\mathcal{P}_{n}$ be the permutation group on $n$ elements, and let $\left\{\psi_{n}\right\}$ be a basis for space $\mathcal{H}$. For each $\sigma \in \mathcal{P}_{n}$, we define an operator (which we also denote by $\sigma$ ) on basis elements $\mathcal{H}^{n}$ by $\sigma\left(\varphi_{k_{1}} \otimes \varphi_{k_{2}} \otimes \cdots \otimes \varphi_{k_{n}}\right)=\varphi_{k_{\sigma(1)}} \otimes \varphi_{k_{\sigma(2)}} \otimes \cdots \otimes \varphi_{k_{\sigma(n)}}$. The operator $\sigma$ extends by linearity to a bounded operator (of norm one) on space $\mathcal{H}^{n}$, so we can define $S_{n}=\frac{1}{n !} \sum_{\sigma \in \mathcal{P}_{n}} \sigma$. It is an easy exercise to show that, the operator $S_{n}$ is the operator of orthogonal projection: $S_{n}^{2}=S_{n}$, and $S_{n}^{*}=S_{n}$. The range of $S_{n}$ is called $n$-fold symmetric tensor product of $\mathcal{H}$. In the case, where $\mathcal{H}=L_{2}(R)$ and $\mathcal{H}^{n}=L_{2}(R) \otimes L_{2}(R) \otimes \cdots \otimes L_{2}(R)=L_{2}\left(R^{n}\right)$, $S_{n} \mathcal{H}^{n}$ is just the subspace of $L_{2}\left(R^{n}\right)$, of all functions, left invariant under any permutation of the variables. We now define $\mathcal{F}_{s}(\mathcal{H})=\bigoplus_{n=0}^{\infty} S_{n} \mathcal{H}^{n}$. The space $\mathcal{F}_{s}(\mathcal{H})$ is called the symmetric Fock space over $\mathcal{H}$, or Boson Fock space over $\mathcal{H}$.

Let $\varepsilon($.$) is function from \mathcal{P}_{n}$ to $\{1,-1\}$, which is one on even permutations and minus one on odd permutations. Define $A_{n}=\frac{1}{n !} \sum_{\sigma \in \mathcal{P}_{n}} \varepsilon(\sigma) \sigma$, then $A_{n}$ is an orthogonal projector on $\mathcal{H}^{n} . A_{n} \mathcal{H}^{n}$ is called the $n$-fold antisymmetric tensor product of $\mathcal{H}$. In the case where $\mathcal{H}=L_{2}(R), A_{n} \mathcal{H}^{n}$ is just the subspace of $L_{2}\left(R^{n}\right)$ consisting of those functions odd under interchange of two coordinates. The subspace $\mathcal{F}_{a}(\mathcal{H})=\bigoplus_{n=0}^{\infty} A_{n} \mathcal{H}^{n}$ is called the antisymmetric Fock space over $\mathcal{H}$, or the Fermion Fock space over $\mathcal{H}$.

\section{Third Doublet State}

In the system exists five type doublet states. The doublet state corresponds to the basis functions ${ }^{1} d_{m, n, r, t, l \in Z^{v}}^{1 / 2}=a_{m, \downarrow}^{+} a_{n, \downarrow}^{+} a_{r, \uparrow}^{+} a_{t, \uparrow}^{+} a_{l, \uparrow}^{+} \varphi_{0} \quad$ and

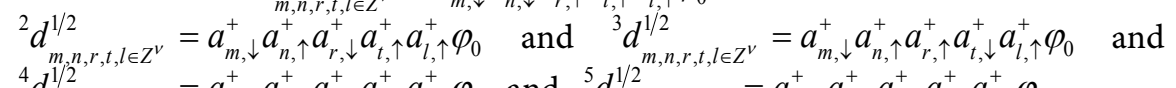
${ }^{4} d_{m, n, r, t, l \in Z^{v}}^{m, n, r, t, l \in Z^{1 / 2}}=a_{m, \downarrow}^{+} a_{n, \uparrow}^{+} a_{r, \uparrow}^{+} a_{t, \uparrow}^{+} a_{l, \downarrow}^{+} \varphi_{0}$ and ${ }^{5} d_{m, n, r, t, l \in Z^{v}}^{1 / m, n, r, t, l \in Z^{\nu}}=a_{m, \uparrow}^{+} a_{n, \downarrow}^{+} a_{r, \downarrow}^{+} a_{t, \uparrow}^{+} a_{l, \uparrow}^{+} \varphi_{0}$.

The subspace ${ }^{3} \mathcal{H}_{1 / 2}^{d}$, corresponding to the third five-electron doublet state is the set of all vectors of the forms

${ }^{3} \psi_{1 / 2}^{d}=\sum_{m, n, r, t, l \in Z^{v}} f(m, n, r, t, l)^{3} d_{m, n, r, t, l \in Z^{\nu}}^{1 / 2}, f \in l_{2}^{a s}$, where $l_{2}^{a s}$ is the subspace of antisymmetric functions in the space $l_{2}\left(\left(Z^{v}\right)^{5}\right)$.

The restriction ${ }^{3} H_{1 / 2}^{d}$ of $H$ to the subspace ${ }^{3} \mathcal{H}_{1 / 2}^{d}$, is called the five-electron third doublet state operator.

Theorem 1. The subspace ${ }^{3} \mathcal{H}_{1 / 2}^{d}$ is invariant under the operator $H$, and the operator ${ }^{3} H_{1 / 2}^{d}$ is a bounded self-adjoint operator. It generates a bounded self-adjoint operator ${ }^{3} \bar{H}_{1 / 2}^{d}$, acting in the space $l_{2}^{a s}$ as

$$
\begin{aligned}
& \left({ }^{3} \bar{H}_{1 / 2}^{d} f\right)(m, n, r, t, l) \\
& =5 \operatorname{Af}(m, n, r, t, l)+B \sum_{\tau}[f(m+\tau, n, r, t, l)+f(m, n+\tau, r, t, l) \\
& \quad+f(m, n, r+\tau, t, l)+f(m, n, r, t+\tau, l)+f(m, n, r, t, l+\tau)] \\
& \quad+U\left[\delta_{m, n}+\delta_{m, r}+\delta_{m, l}+\delta_{n, t}+\delta_{r, t}+\delta_{t, l}\right] f(m, n, r, t, l),
\end{aligned}
$$


where $\delta_{k, j}$ is the Kronecker symbol. The operator ${ }^{3} H_{1 / 2}^{d}$ acts on a vector ${ }^{3} \psi_{1 / 2}^{d} \in{ }^{3} \mathcal{H}_{1 / 2}^{d}$ as

$$
\left.{ }^{3} H_{1 / 2}^{d}{ }^{3} \psi_{1 / 2}^{d}=\sum_{m, n, r, t, l \in Z^{\nu}}\left({ }^{3} \bar{H}_{1 / 2}^{d} f\right)(m, n, r, t, l)\right)^{3} d_{m, n, r, t, l \in Z^{\nu}}^{1 / 2} .
$$

Proof. We act with the Hamiltonian $H$ on vectors $\psi \in{ }^{3} \mathcal{H}_{1 / 2}^{d}$ using the standard anticommutation relations between electron creation and annihilation operators at lattice sites, $\left\{a_{m, \gamma}, a_{n, \beta}^{+}\right\}=\delta_{m, n} \delta_{\gamma, \beta},\left\{a_{m, \gamma}, a_{n, \beta}\right\}=\left\{a_{m, \gamma}^{+}, a_{n, \beta}^{+}\right\}=\theta$, and also take into account that $a_{m, \gamma} \varphi_{0}=\theta$, where $\theta$ is the zero element of ${ }^{3} \mathcal{H}_{1 / 2}^{d}$. This yields the statement of the theorem.

Lemma 1. The spectrum of the operators ${ }^{3} H_{1 / 2}^{d}$ and ${ }^{3} \bar{H}_{1 / 2}^{d}$ coincide.

Proof. Because ${ }^{3} H_{1 / 2}^{d}$ and ${ }^{3} \bar{H}_{1 / 2}^{d}$ are bounded self-adjoint operators, it follows from the Weyl criterion (see, for example, [14], Ch. VII, \$14) that there exist a sequence of vectors $\psi_{n}$ such that

$$
\psi_{n}=\sum_{p, q, r, t, s} f_{n}(p, q, r, t, s) a_{p, \downarrow}^{+} a_{q, \uparrow}^{+} a_{r, \uparrow}^{+} a_{t, \downarrow}^{+} a_{s, \uparrow}^{+} \varphi_{0},\left\|\psi_{n}\right\|=1,
$$

and

$$
\lim _{n \rightarrow \infty}\left\|{ }^{3} H_{1 / 2}^{d} \psi_{n}-\lambda \psi_{n}\right\|=0,
$$

where $\lambda \in \sigma\left({ }^{3} H_{1 / 2}^{d}\right)$. On the other hand,

$$
\begin{aligned}
& \left\|{ }^{3} H_{1 / 2}^{d} \psi_{n}-\lambda \psi_{n}\right\|^{2}=\left({ }^{3} H_{1 / 2}^{d} \psi_{n}-\lambda \psi_{n},{ }^{3} H_{1 / 2}^{d} \psi_{n}-\lambda \psi_{n}\right) \\
= & \sum_{p, q, r, t, s}\left\|{ }^{3} \bar{H}_{1 / 2}^{d} f_{n}(p, q, r, t, s)-\lambda f_{n}(p, q, r, t, s)\right\|^{2} \\
& \times\left(a_{p, \downarrow}^{+} a_{q, \uparrow}^{+} a_{r, \uparrow}^{+} a_{t, \downarrow}^{+} a_{s, \uparrow}^{+} \varphi_{0}, a_{p, \downarrow}^{+} a_{q, \uparrow}^{+} a_{r, \uparrow}^{+} a_{t, \downarrow}^{+} a_{s, \uparrow}^{+} \varphi_{0}\right) \\
= & \left\|{ }^{3} \bar{H}_{1 / 2}^{d} F_{n}-\lambda F_{n}\right\|^{2} \times\left(a_{p, \downarrow} a_{q, \uparrow} a_{r, \uparrow} a_{t, \downarrow} a_{s, \uparrow} a_{p, \downarrow}^{+} a_{q, \uparrow}^{+} a_{r, \uparrow}^{+} a_{t, \downarrow}^{+} a_{s, \uparrow}^{+} \varphi_{0}, \varphi_{0}\right) \\
= & \left\|\left({ }^{3} \bar{H}_{1 / 2}^{d}-\lambda\right) F_{n}\right\|^{2}\left(\varphi_{0}, \varphi_{0}\right)=\left\|\left({ }^{3} \bar{H}_{1 / 2}^{d}-\lambda\right) F_{n}\right\|^{2} \rightarrow 0,
\end{aligned}
$$

$n \rightarrow \infty$. Here $F_{n}=\left(f_{n}(p, q, r, t, s)\right)_{p, q, r, t, s \in Z^{v}}$ and

$\left\|F_{n}\right\|^{2}=\sum_{p, q, r, t, s}\left|f_{n}(p, q, r, t, s)\right|^{2}=\left\|\psi_{n}\right\|^{2}=1$. It follows that $\lambda \in \sigma\left({ }^{3} \bar{H}_{1 / 2}^{d}\right)$. Consequently, $\sigma\left({ }^{3} H_{1 / 2}^{d}\right) \subset \sigma\left({ }^{3} \bar{H}_{1 / 2}^{d}\right)$. Conversely, let $\bar{\lambda} \in \sigma\left({ }^{3} \bar{H}_{1 / 2}^{d}\right)$. Again by the Weyl criterion, there then exist a sequence $F_{n}$ such that

$$
\left\|F_{n}\right\|=\sqrt{\sum_{p, q, r, t, s}\left|f_{n}(p, q, r, t, s)\right|^{2}}=1
$$

and

$$
\left\|{ }^{3} \bar{H}_{1 / 2}^{d} F_{n}-\bar{\lambda} F_{n}\right\| \rightarrow 0
$$

as $n \rightarrow \infty$.

Setting $\psi_{n}=\sum_{p, q, r, t, s} f_{n}(p, q, r, t, s) a_{p, \downarrow}^{+} a_{q, \uparrow}^{+} a_{r, \uparrow}^{+} a_{t, \downarrow}^{+} a_{s, \uparrow}^{+} \varphi_{0}$, we have $\left\|\psi_{n}\right\|=\left\|F_{n}\right\|=1$ and $\left\|{ }^{3} \bar{H}_{1 / 2}^{d} F_{n}-\bar{\lambda} F_{n}\right\|=\left\|{ }^{3} H_{1 / 2}^{d} \psi_{n}-\bar{\lambda} \psi_{n}\right\|$. This, together with Formula (6) and the Weyl criterion, implies that $\bar{\lambda} \in \sigma\left({ }^{3} H_{1 / 2}^{d}\right)$, and hence $\sigma\left({ }^{3} \bar{H}_{1 / 2}^{d}\right) \subset \sigma\left({ }^{3} H_{1 / 2}^{d}\right)$. These two relations imply that $\sigma\left({ }^{3} \bar{H}_{1 / 2}^{d}\right)=\sigma\left({ }^{3} H_{1 / 2}^{d}\right)$.

We let $\mathcal{F}$ denote the Fourier transform: $\mathcal{F}: l_{2}\left(\left(Z^{v}\right)^{5}\right) \rightarrow L_{2}\left(\left(T^{v}\right)^{5}\right) \equiv{ }^{3} \tilde{\mathcal{H}}_{1 / 2}^{d}$, 
where $T^{v}$ is a $v$-dimensional torus with the normalized Lebesgue measure $d \lambda: \lambda\left(T^{v}\right)=1$.

We set ${ }^{3} \tilde{H}_{1 / 2}^{d}=\mathcal{F}^{3} \bar{H}_{1 / 2}^{d} \mathcal{F}^{-1}$. In the quasimomentum representation, the operator ${ }^{3} \tilde{H}_{1 / 2}^{d}$ acts in the Hilbert space $L_{2}^{a s}\left(\left(T^{v}\right)^{5}\right)$ as

$$
\begin{aligned}
&{ }^{3} \tilde{H}_{1 / 2}^{d}{ }^{3} \psi_{1 / 2}^{d} \\
&=\left\{5 A+2 B \sum_{i=1}^{v}\left[\cos \lambda_{i}+\cos \mu_{i}+\cos \gamma_{i}+\cos \theta_{i}+\cos \eta_{i}\right]\right\} f(\lambda, \mu, \gamma, \theta, \eta) \\
&+U \int_{T^{v}}[f(s, \lambda+\mu-s, \gamma, \theta, \eta)+f(s, \mu, \lambda+\gamma-s, \theta, \eta) \\
&+f(s, \mu, \gamma, \theta, \lambda+\eta-s)+f(\lambda, s, \gamma, \mu+\theta-s, \eta) \\
&+f(\lambda, \mu, s, \gamma+\theta-s, \eta)+f(\lambda, \mu, \gamma, s, \theta+\eta-s)] \mathrm{d} s,
\end{aligned}
$$

where $L_{2}^{a s}\left(\left(T^{v}\right)^{5}\right)$ is the subspace of antisymmetric functions in $L_{2}\left(\left(T^{v}\right)^{5}\right)$.

Using tensor products of Hilbert spaces and tensor products of operators in Hilbert spaces [15], we can verify that the operator ${ }^{3} \tilde{H}_{1 / 2}^{d}$ can be represented in the form

$$
{ }^{3} \tilde{H}_{1 / 2}^{d}{ }^{3} \psi_{1 / 2}^{d}=\tilde{H}_{2}^{1}(\lambda, \mu) \otimes I \otimes I+I \otimes \tilde{H}_{2}^{2}(\gamma, \theta) \otimes I+I \otimes I \otimes \tilde{H}_{2}^{3}(\theta, \eta)
$$

where

$$
\begin{aligned}
\left(\tilde{H}_{2}^{1} f\right)(\lambda, \mu)= & \left\{2 A+2 B \sum_{i=1}^{v}\left(\cos \lambda_{i}+\cos \mu_{i}\right)\right\} f(\lambda, \mu) \\
& +U \int_{T^{v}} f(s, \lambda+\mu-s) \mathrm{d} s+U \int_{T^{v}} f(s, \gamma+\theta-s) \mathrm{d} s \\
\left(\tilde{H}_{2}^{2} f\right)(\gamma, \theta)= & \left\{2 A+2 B \sum_{i=1}^{v}\left(\cos \gamma_{i}+\cos \theta_{i}\right)\right\} f(\gamma, \theta) \\
& -U \int_{T^{v}} f(s, \lambda+\gamma-s) \mathrm{d} s-U \int_{T^{v}} f(s, \lambda+\eta-s) \mathrm{d} s \\
\left(\tilde{H}_{2}^{3} f\right)(\theta, \eta)= & \left\{A+2 B \sum_{i=1}^{v} \cos \eta_{i}\right\} f(\theta, \eta)+U \int_{T^{v}} f(s, \theta+\eta-s) \mathrm{d} s \\
& -U \int_{T^{v}} f(s, \mu+\theta-s) \mathrm{d} s,
\end{aligned}
$$

and $I$ is the unit operator.

Taking into account that $\lambda, \mu, \gamma, \theta, \eta \in T^{v}$, we can express the action of operators $\tilde{H}_{2}^{1}, \tilde{H}_{2}^{2}$ and $\tilde{H}_{2}^{3}$ in the form

$$
\begin{aligned}
&\left(\tilde{H}_{2}^{1} f\right)(\lambda, \mu)=\left\{2 A+2 B \sum_{i=1}^{v}\left(\cos \lambda_{i}+\cos \mu_{i}\right)\right\} f(\lambda, \mu) \\
&+2 U \int_{T^{v}} f(s, \lambda+\mu-s) \mathrm{d} s, \\
&\left(\tilde{H}_{2}^{2} f\right)(\gamma, \theta)=\left\{2 A+2 B \sum_{i=1}^{v}\left(\cos \gamma_{i}+\cos \theta_{i}\right)\right\} f(\gamma, \theta) \\
&-2 U \int_{T^{v}} f(s, \gamma+\theta-s) \mathrm{d} s, \\
&\left(\tilde{H}_{2}^{3} f\right)(\theta, \eta)=\left\{A+2 B \sum_{i=1}^{v} \cos \eta_{i}\right\} f(\theta, \eta) .
\end{aligned}
$$

The spectrum of the operator $A \otimes I+I \otimes B$, where $A$ and $B$ are densely defined bounded linear operators, was studied in [16] [17] [18]. Explicit formulas 
were given there that express the essential spectrum $\sigma_{\text {ess }}(A \otimes I+I \otimes B)$ and discrete spectrum $\sigma_{\text {disc }}(A \otimes I+I \otimes B)$ of operator $A \otimes I+I \otimes B$ in terms of the spectrum $\sigma(A)$ and the discrete spectrum $\sigma_{\text {disc }}(A)$ of $A$ and in terms of the spectrum $\sigma(B)$ and the discrete spectrum $\sigma_{\text {disc }}(B)$ of $B$ :

$$
\begin{aligned}
& \sigma_{\text {disc }}(A \otimes I+I \otimes B) \\
&=\left\{\sigma(A) \backslash \sigma_{\text {ess }}(A)+\sigma(B) \backslash \sigma_{\text {ess }}(B)\right\} \backslash\left\{\left(\sigma_{\text {ess }}(A)+\sigma(B)\right)\right. \\
&\left.\bigcup\left(\sigma(A)+\sigma_{\text {ess }}(B)\right)\right\}, \\
& \sigma_{\text {ess }}(A \otimes I+I \otimes B)=\left(\sigma_{\text {ess }}(A)+\sigma(B)\right) \bigcup\left(\sigma(A)+\sigma_{\text {ess }}(B)\right) .
\end{aligned}
$$

It is clear that $\sigma(A \otimes I+I \otimes B)=\{\lambda+\mu: \lambda \in \sigma(A), \mu \in \sigma(B)\}$.

Therefore, we must investigate the spectrum of the operators $\tilde{H}_{2}^{1}, \tilde{H}_{2}^{2}$ and $\tilde{H}_{2}^{3}$.

Let $\Lambda_{1}=\lambda+\mu, \Lambda_{2}=\gamma+\theta, \Lambda_{3}=\theta+\eta$.

Let the total quasimomentum of the two-electron system $\lambda+\mu=\Lambda_{1}$ be fixed. We let $L_{2}\left(\Gamma_{\Lambda_{1}}\right)$ denote the space of functions that are square integrable on the manifold $\Gamma_{\Lambda_{1}}=\left\{(\lambda, \mu): \lambda+\mu=\Lambda_{1}\right\}$. It is known ([19], chapter II, pp. 63-84, and [15], chapter XIII, paragraph 16, pp. 303-341) that the operator $\tilde{H}_{2}^{1}$ and the space $\tilde{\mathcal{H}}_{2}^{1} \equiv L_{2}\left(\left(T^{v}\right)^{2}\right)$ can be decomposed into a direct integral $\tilde{H}_{2}^{1}=\oplus \int_{T^{v}} \tilde{H}_{2 \Lambda_{1}}^{1} \mathrm{~d} \Lambda_{1}, \quad \tilde{\mathcal{H}}_{2}^{1}=\bigoplus \int_{T^{v}} \tilde{\mathcal{H}}_{2 \Lambda_{1}}^{1} \mathrm{~d} \Lambda_{1} \quad$ of operators $\tilde{H}_{2 \Lambda_{1}}^{1}$ and spaces $\tilde{\mathcal{H}}_{2 \Lambda_{1}}^{1}=L_{2}\left(\Gamma_{\Lambda_{1}}\right)$ such that $\tilde{\mathcal{H}}_{2 \Lambda_{1}}^{1}$ are invariant under $\tilde{H}_{2 \Lambda_{1}}^{1}$ and $\tilde{H}_{2 \Lambda_{1}}^{1}$ act in $\tilde{\mathcal{H}}_{2 \Lambda_{1}}^{1}$ according to the formula

$$
\left(\tilde{H}_{2 \Lambda_{1}}^{1} f_{\Lambda_{1}}\right)(\lambda)=\left\{2 A+4 B \sum_{i=1}^{v} \cos \frac{\Lambda_{1}^{i}}{2} \cos \left(\frac{\Lambda_{1}^{i}}{2}-\lambda_{i}\right)\right\} f_{\Lambda_{1}}(\lambda)+2 U \int_{T^{v}} f_{\Lambda_{1}}(s) \mathrm{d} s,
$$

where $f_{\Lambda_{1}}(x)=f\left(x, \Lambda_{1}-x\right)$.

It is known that the continuous spectrum of $\tilde{H}_{2 \Lambda_{1}}^{1}$ is independent of the parameter $U$ and consists of the intervals

$$
\sigma_{\text {cont }}\left(\tilde{H}_{2 \Lambda_{1}}^{1}\right)=G_{\Lambda_{1}}^{v}=\left[m_{\Lambda_{1}}^{v}, M_{\Lambda_{1}}^{v}\right]=\left[2 A-4 B \sum_{i=1}^{v} \cos \frac{\Lambda_{1}^{i}}{2}, 2 A+4 B \sum_{i=1}^{v} \cos \frac{\Lambda_{1}^{i}}{2}\right] \text {. }
$$

Definition 1. The eigenfunction $\varphi_{\Lambda_{1}} \in L_{2}\left(T^{v} \times T^{v}\right)$ of the operator $\tilde{H}_{2 \Lambda_{1}}^{1}$ corresponding to an eigenvalue $z_{\Lambda_{1}} \notin G_{\Lambda_{1}}^{v}$ is called a bound state (BS) (antibound state $(A B S)$ ) of $\tilde{H}_{2}^{1}$ with the quasi momentum $\Lambda_{1}$, and the quantity $z_{\Lambda_{1}}$ is called the energy of this state.

We consider the operator $K_{\Lambda_{1}}(z)$ acting in the space $\tilde{\mathcal{H}}_{2 \Lambda_{1}}^{1}$ according to the formula

$$
\left(K_{\Lambda_{1}}(z) f_{\Lambda_{1}}\right)(x)=\int_{T^{v}} \frac{2 U}{2 A+4 B \sum_{i=1}^{v} \cos \frac{\Lambda_{1}^{i}}{2} \cos \left(\frac{\Lambda_{1}^{i}}{2}-t_{i}\right)-z} f_{\Lambda_{1}}(t) \mathrm{d} t .
$$

It is a completely continuous operator in $\tilde{\mathcal{H}}_{2 \Lambda_{1}}^{1}$ for $z \notin G_{\Lambda_{1}}^{v}$.

$$
\text { We set } D_{\Lambda_{1}}^{v}(z)=1+2 U \int_{T^{v}} \frac{\mathrm{d} s_{1} \mathrm{~d} s_{2} \cdots \mathrm{d} s_{v}}{2 A+4 B \sum_{i=1}^{v} \cos \frac{\Lambda_{1}^{i}}{2} \cos \left(\frac{\Lambda_{1}^{i}}{2}-s_{i}\right)-z} \text {. }
$$


Lemma 2. A number $z=z_{0} \notin G_{\Lambda_{1}}^{v}$ is an eigenvalue of the operator $\tilde{H}_{2 \Lambda_{1}}^{1}$ if and only if it is a zero of the function $D_{\Lambda_{1}}^{v}(z)$, i.e., $D_{\Lambda_{1}}^{v}\left(z_{0}\right)=0$.

Proof. Let the number $z=z_{0} \notin G_{\Lambda_{1}}^{v}$ be an eigenvalue of the operator $\tilde{H}_{2 \Lambda_{1}}^{1}$, and $\varphi_{\Lambda_{1}}(x)$ be the corresponding eigenfunction, i.e.,

$$
\left\{2 A+4 B \sum_{i=1}^{v} \cos \frac{\Lambda_{1}^{i}}{2} \cos \left(\frac{\Lambda_{1}^{i}}{2}-x_{i}\right)\right\} \varphi_{\Lambda_{1}}(x)+2 U \int_{T^{\nu}} \varphi_{\Lambda_{1}}(s) \mathrm{d} s=z_{0} \varphi_{\Lambda_{1}}(x) .
$$

Let $\psi_{\Lambda_{1}}(x)=\left[2 A+4 B \sum_{i=1}^{v} \cos \frac{\Lambda_{1}^{i}}{2} \cos \left(\frac{\Lambda_{1}^{i}}{2}-x_{i}\right)-z\right] \varphi_{\Lambda_{1}}(x)$. Then

$$
\psi_{\Lambda_{1}}(x)+\int_{T^{V}} \frac{2 U}{2 A+4 B \sum_{i=1}^{v} \cos \frac{\Lambda_{1}^{i}}{2} \cos \left(\frac{\Lambda_{1}^{i}}{2}-x_{i}\right)-z} \psi_{\Lambda_{1}}(t) \mathrm{d} t=0,
$$

i.e., the number $\mu=1$ is an eigenvalue of the operator $K_{\Lambda_{1}}(z)$. It then follows that $D_{\Lambda_{1}}^{v}\left(z_{0}\right)=0$.

Now let $z=z_{0}$ be a zero of the function $D_{\Lambda_{1}}^{v}(z)$, i.e., $D_{\Lambda_{1}}^{v}\left(z_{0}\right)=0$. It follows from the Fredholm theorem than the homogeneous equation

$$
\psi_{\Lambda_{1}}(x)+2 U \int_{T^{v}} \frac{\psi_{\Lambda_{1}}(s) \mathrm{d} s_{1} \cdots \mathrm{d} s_{v}}{2 A+4 B \sum_{i=1}^{v} \cos \frac{\Lambda_{1}^{i}}{2} \cos \left(\frac{\Lambda_{1}^{i}}{2}-s_{i}\right)-z} \mathrm{~d} s=0
$$

has a nontrivial solution. This means that the number $z=z_{0}$ is an eigenvalue of the operator $\tilde{H}_{2 \Lambda_{1}}^{1}$.

We consider the one-dimensional case.

Theorem 2. 1). At $v=1$ and $U<0$, and all values of parameters of the Hamiltonian, the operator $\tilde{H}_{2 \Lambda_{1}}^{1}$ has a unique eigenvalue $z_{1}=2 A-2 \sqrt{U^{2}+4 B^{2} \cos ^{2} \frac{\Lambda_{1}}{2}}$, that is below the continuous spectrum of $\tilde{H}_{2 \Lambda_{1}}^{1}$, i.e., $z_{1}<m_{\Lambda_{1}}^{1}$.

2). At $v=1$ and $U>0$, and all values of parameters of the Hamiltonian, the operator $\tilde{H}_{2 \Lambda_{1}}^{1}$ has a unique eigenvalue $\tilde{z}_{1}=2 A+2 \sqrt{U^{2}+4 B^{2} \cos ^{2} \frac{\Lambda_{1}}{2}}$, that is above the continuous spectrum of $\tilde{H}_{2 \Lambda_{1}}^{1}$, i.e., $\tilde{z}_{1}>M_{\Lambda_{1}}^{1}$.

Proof. If $U<0$, then in the one-dimensional case, the function $D_{\Lambda_{1}}^{v}(z)$ is a monotone decreasing function on $\left(-\infty, m_{\Lambda_{1}}^{1}\right)$ and $\left(M_{\Lambda_{1}}^{1}, \infty\right)$, i.e., outside the continuous spectrum domain of the operator $\tilde{H}_{2 \Lambda_{1}}^{1}$. For $z<m_{\Lambda_{1}}^{1}$ the function $D_{\Lambda_{1}}^{v}(z)$ decreases from 1 to $-\infty, D_{\Lambda_{1}}^{v}(z) \rightarrow 1$ as $z \rightarrow-\infty, D_{\Lambda_{1}}^{v}(z) \rightarrow-\infty$ as $z \rightarrow m_{\Lambda_{1}}^{1}-0$. Therefore, below the value $m_{\Lambda_{1}}^{1}$, the function $D_{\Lambda_{1}}^{v}(z)$ has a single zero at the point $z=z_{1}=2 A-2 \sqrt{U^{2}+4 B^{2} \cos ^{2} \frac{\Lambda_{1}}{2}}<m_{\Lambda_{1}}^{1}$. For $z>M_{\Lambda_{1}}^{1}$, and $U<0$, the function $D_{\Lambda_{1}}^{v}(z)$ decreases from $+\infty$ to $1, D_{\Lambda_{1}}^{v}(z) \rightarrow+\infty$ as $z \rightarrow M_{\Lambda_{1}}^{1}+0, D_{\Lambda_{1}}^{v}(z) \rightarrow 1$ as $z \rightarrow+\infty$. Therefore, above the value $M_{\Lambda_{1}}^{1}$, the function $D_{\Lambda_{1}}^{v}(z)$ cannot vanish. 
If $U>0$, then the function $D_{\Lambda_{1}}^{v}(z)$ is an monotone increasing function on $\left(-\infty, m_{\Lambda_{1}}^{1}\right)$ and $\left(M_{\Lambda_{1}}^{1}, \infty\right)$, i.e., outside the continuous spectrum domain of the operator $\tilde{H}_{2 \Lambda_{1}}^{1}$. For $z<m_{\Lambda_{1}}^{1}$ the function $D_{\Lambda_{1}}^{v}(z)$ increases from 1 to $+\infty$, $D_{\Lambda_{1}}^{v}(z) \rightarrow 1$ as $z \rightarrow-\infty, D_{\Lambda_{1}}^{v}(z) \rightarrow+\infty$ as $z \rightarrow m_{\Lambda_{1}}^{1}-0$. Therefore, below the value $m_{\Lambda_{1}}^{1}$, the function $D_{\Lambda_{1}}^{v}(z)$ cannot vanish.

For $z>M_{\Lambda_{1}}^{1}$, and $U>0$, the function $D_{\Lambda_{1}}^{v}(z)$ increases from $-\infty$ to 1 , $D_{\Lambda_{1}}^{v}(z) \rightarrow-\infty$ as $z \rightarrow M_{\Lambda_{1}}^{1}+0, \quad D_{\Lambda_{1}}^{v}(z) \rightarrow 1$ as $z \rightarrow+\infty$. Therefore, above the value $M_{\Lambda_{1}}^{1}$, the function $D_{\Lambda_{1}}^{v}(z)$ has a single zero at the point $\tilde{z}_{1}=2 A+2 \sqrt{U^{2}+4 B^{2} \cos ^{2} \frac{\Lambda_{1}}{2}}>M_{\Lambda_{1}}^{1}$.

In the two-dimensional case, we have analogous results.

If $U>0$, then the function $D_{\Lambda_{1}}^{2}$ is an increasing monotone function on $\left(-\infty, m_{\Lambda_{1}}^{2}\right)$ and $\left(M_{\Lambda_{1}}^{2}, \infty\right)$, i.e., outside the continuous spectrum domain of the operator $\tilde{H}_{2 \Lambda_{1}}^{1}$. For $z<m_{\Lambda_{1}}^{2}$ the function $D_{\Lambda_{1}}^{v}(z)$ increases from 1 to $+\infty$, $D_{\Lambda_{1}}^{v}(z) \rightarrow 1$ as $z \rightarrow-\infty, D_{\Lambda_{1}}^{v}(z) \rightarrow+\infty$ as $z \rightarrow m_{\Lambda_{1}}^{2}-0$. Therefore, below the value $m_{\Lambda_{1}}^{2}$, the function $D_{\Lambda_{1}}^{v}(z)$ cannot vanish. For $z>M_{\Lambda_{1}}^{2}$ and $U>0$, the function $D_{\Lambda_{1}}^{v}(z)$ increases from $-\infty$ to $1, D_{\Lambda_{1}}^{v}(z) \rightarrow 1$ as $z \rightarrow+\infty$, $D_{\Lambda_{1}}^{v}(z) \rightarrow-\infty$ as $z \rightarrow M_{\Lambda_{1}}^{2}+0$. Therefore, above the value $M_{\Lambda_{1}}^{2}$, the function $D_{\Lambda_{1}}^{v}(z)$ has a single zero at the point $\tilde{z}_{1}>M_{\Lambda_{1}}^{2}$.

If $U<0$, for $z>M_{\Lambda_{1}}^{2}$, then the function $D_{\Lambda_{1}}^{2}(z)$ decreases from $+\infty$ to $1, \quad D_{\Lambda_{1}}^{v}(z) \rightarrow 1$ as $z \rightarrow+\infty, \quad D_{\Lambda_{1}}^{v}(z) \rightarrow+\infty$ as $z \rightarrow M_{\Lambda_{1}}^{2}+0$. Therefore, above the value $M_{\Lambda_{1}}^{2}$, the function $D_{\Lambda_{1}}^{v}(z)$ cannot vanish. If $U<0$, for $z<m_{\Lambda_{1}}^{2}$, then the function $D_{\Lambda_{1}}^{2}(z)$ decreases from 1 to $-\infty, D_{\Lambda_{1}}^{v}(z) \rightarrow 1$ as $z \rightarrow-\infty, D_{\Lambda_{1}}^{v} \rightarrow-\infty$ as $z \rightarrow m_{\Lambda_{1}}^{2}-0$. Therefore, below the value $m_{\Lambda_{1}}^{2}$, the function $D_{\Lambda_{1}}^{v}(z)$ has a single zero at the point $z_{1}<m_{\Lambda_{1}}^{2}$.

We consider three-dimensional case. Denote

$m=\int_{T^{3}} \frac{\mathrm{d} s_{1} \mathrm{~d} s_{2} \mathrm{~d} s_{3}}{\sum_{i=1}^{3} \cos \frac{\Lambda_{1}^{i}}{2}\left(1+\cos \left(\frac{\Lambda_{1}^{i}}{2}-s_{i}\right)\right)}$.

For $U<0$, and $U<-\frac{2 B}{m}$, below the continuous spectrum of the operator $\tilde{H}_{2 \Lambda_{1}}^{1}$, the function $D_{\Lambda_{1}}^{3}(z)$ has a single zero at the point $z=z_{1}<m_{\Lambda_{1}}^{3}$. For $U<0$, and $-\frac{2 B}{m} \leq U<0$, below the continuous spectrum of the operator $\tilde{H}_{2 \Lambda_{1}}^{1}$, the function $D_{\Lambda_{1}}^{3}(z)$ cannot vanish.

Denote $M=\int_{T^{3}} \frac{\mathrm{d} s_{1} \mathrm{~d} s_{2} \mathrm{~d} s_{3}}{\sum_{i=1}^{3} \cos \frac{\Lambda_{1}^{i}}{2}\left(1-\cos \left(\frac{\Lambda_{1}^{i}}{2}-s_{i}\right)\right)}$.

For $U>0$, and $U>\frac{2 B}{M}$, above the continuous spectrum of the operator $\tilde{H}_{2 \Lambda_{1}}^{1}$, the function $D_{\Lambda_{1}}^{3}(z)$ has a single zero at the point $\tilde{z}_{1}>M_{\Lambda_{1}}^{3}$. For $0<U \leq \frac{2 B}{M}$, above the continuous spectrum of the operator $\tilde{H}_{2 \Lambda_{1}}^{1}$, the function $D_{\Lambda_{1}}^{3}(z)$ cannot vanish.

Now we investigated the spectrum of the operator $\tilde{H}_{2 \Lambda_{2}}^{2}$, i.e., the operator 


$$
\left(\tilde{H}_{2 \Lambda_{2}}^{2} f_{\Lambda_{2}}\right)(\gamma)=\left\{2 A+4 B \sum_{i=1}^{v} \cos \frac{\Lambda_{2}^{i}}{2} \cos \left(\frac{\Lambda_{2}^{i}}{2}-\gamma_{i}\right)\right\} f_{\Lambda_{2}}(\gamma)-2 U \int_{T^{v}} f_{\Lambda_{2}}(s) \mathrm{d} s .
$$

It is known that the continuous spectrum of the operator $\tilde{H}_{2 \Lambda_{2}}^{2}$ is independent of $U$ and coincides with the segment

$$
\sigma_{\text {cont }}\left(\tilde{H}_{2 \Lambda_{2}}^{2}\right)=\left[2 A-4 B \sum_{i=1}^{v} \cos \frac{\Lambda_{2}^{i}}{2}, 2 A+4 B \sum_{i=1}^{v} \cos \frac{\Lambda_{2}^{i}}{2}\right]=G_{\Lambda_{2}}^{v} .
$$

We let $L_{2}\left(\Gamma_{\Lambda_{2}}\right)$ denote the space of functions that are square integrable on the manifold $\Gamma_{\Lambda_{2}}=\left\{(\gamma, \theta): \gamma+\theta=\Lambda_{2}\right\}$. That the operator $\tilde{H}_{2}^{2}$ and the space $\tilde{\mathcal{H}}_{2}^{2} \equiv L_{2}\left(\left(T^{v}\right)^{2}\right)$ can be decomposed into a direct integral $\tilde{H}_{2}^{2}=\bigoplus \int_{T^{v}} \tilde{H}_{2 \Lambda_{2}}^{2} \mathrm{~d} \Lambda_{2}$, $\tilde{\mathcal{H}}_{2}^{2}=\oplus \int_{T^{\nu}} \tilde{\mathcal{H}}_{2 \Lambda_{2}}^{2} \mathrm{~d} \Lambda_{2}$. Each operator $\tilde{H}_{2 \Lambda_{2}}^{2}$ acts in $\tilde{\mathcal{H}}_{2 \Lambda_{2}}^{2}=L_{2}\left(\Gamma_{\Lambda_{2}}\right)$ as $\left(\tilde{H}_{2 \Lambda_{2}}^{2} f_{\Lambda_{2}}\right)(\gamma)=\left\{2 A+4 B \sum_{i=1}^{v} \cos \frac{\Lambda_{2}^{i}}{2} \cos \left(\frac{\Lambda_{2}^{i}}{2}-\gamma_{i}\right)\right\} f_{\Lambda_{2}}(\gamma)-2 U \int_{T^{v}} f_{\Lambda_{2}}(s) \mathrm{d} s$, where $f_{\Lambda_{2}}(x)=f\left(x, \Lambda_{2}-x\right)$.

$$
\text { We set } D_{\Lambda_{2}}^{v}(z)=1-2 U \int_{T^{v}} \frac{\mathrm{d} s_{1} \mathrm{~d} s_{2} \cdots \mathrm{d} s_{v}}{2 A+4 B \sum_{i=1}^{v} \cos \frac{\Lambda_{2}^{i}}{2} \cos \left(\frac{\Lambda_{2}^{i}}{2}-s_{i}\right)-z} \text {. }
$$

The analogue of the Lemma 2 holds for the in this case. We consider the one-dimensional case.

Theorem 3. 1) At $v=1$ and $U>0$ and for all values of the parameter of the Hamiltonian, the operator $\tilde{H}_{2 \Lambda_{2}}^{2}$ has a unique eigenvalue $z=z_{2}=2 A-2 \sqrt{U^{2}+4 B^{2} \cos ^{2} \frac{\Lambda_{2}}{2}}$, that is below the continuous spectrum of operator $\tilde{H}_{2 \Lambda_{2}}^{2}$, i.e., $z_{2}<m_{\Lambda_{2}}^{1}$.

2) At $v=1$ and $U<0$ and for all values of the parameter of the Hamiltonian, the operator $\tilde{H}_{2 \Lambda_{2}}^{2}$ has a unique eigenvalue $z=\tilde{z}_{2}=2 A+2 \sqrt{U^{2}+4 B^{2} \cos ^{2} \frac{\Lambda_{2}}{2}}$, that is above the continuous spectrum of operator $\tilde{H}_{2 \Lambda_{2}}^{2}$, i.e., $\tilde{z}_{2}>M_{\Lambda_{2}}^{1}$.

The Theorem 3 is proved totally similarly to Theorem 2 .

In the two-dimensional case, we have analogous results.

If $U>0$, then the equation $D_{\Lambda_{2}}^{2}(z)=0$ has a unique solution $z_{2}<m_{\Lambda_{2}}^{2}$, below the continuous spectrum of the operator $\tilde{H}_{2 \Lambda_{2}}^{2}$. If $U<0$, then the equation $D_{\Lambda_{2}}^{2}(z)=0$ has a unique solution $\tilde{z}_{2}>M_{\Lambda_{2}}^{2}$, above the continuous spectrum of the operator $\tilde{H}_{2 \Lambda_{2}}^{2}$.

We consider three-dimensional case. Denote

$$
m=\int_{T^{3}} \frac{\mathrm{d} s_{1} \mathrm{~d} s_{2} \mathrm{~d} s_{3}}{\sum_{i=1}^{3} \cos \frac{\Lambda_{2}^{i}}{2}\left(1+\cos \left(\frac{\Lambda_{2}^{i}}{2}-s_{i}\right)\right)} .
$$

For $U>0$, and $U>\frac{2 B}{m}$, below of the continuous spectrum of the operator $\tilde{H}_{2 \Lambda_{2}}^{2}$, the function $D_{\Lambda_{2}}^{3}(z)$ has a single zero at the point $z=z_{2}<m_{\Lambda_{2}}^{3}$. For 
$U>0$, and $0<U \leq \frac{2 B}{m}$, below the continuous spectrum of the operator $\tilde{H}_{2 \Lambda_{2}}^{2}$, the function $D_{\Lambda_{2}}^{3}(z)$ cannot vanish.

Denote $M=\int_{T^{3}} \frac{\mathrm{d} s_{1} \mathrm{~d} s_{2} \mathrm{~d} s_{3}}{\sum_{i=1}^{3} \cos \frac{\Lambda_{2}^{i}}{2}\left(1-\cos \left(\frac{\Lambda_{2}^{i}}{2}-s_{i}\right)\right)}$.

For $U<0$, and $U<-\frac{2 B}{M}$, above of the continuous spectrum of the operator $\tilde{H}_{2 \Lambda_{2}}^{2}$, the function $D_{\Lambda_{2}}^{3}(z)$ has a single zero at the point $z=\tilde{z}_{2}>M_{\Lambda_{2}}^{3}$. For $U<0$, and $-\frac{2 B}{M} \leq U<0$, above the continuous spectrum of the operator $\tilde{H}_{2 \Lambda_{2}}^{2}$, the function $D_{\Lambda_{2}}^{3}(z)$ cannot vanish.

Now we investigated the spectrum of the operator $\tilde{H}_{\Lambda_{3}}^{3}$.

$$
\left(\tilde{H}_{\Lambda_{3}}^{3} f_{\Lambda_{3}}\right)(\theta)=\left\{A+2 B \sum_{i=1}^{v} \cos \left(\Lambda_{3}-\theta_{i}\right)\right\} f_{\Lambda_{3}}(\theta) .
$$

It is obvious that the spectrum of operator $\tilde{H}_{\Lambda_{3}}^{3}$ is purely continuous and coincides with the value set of the function $h_{\Lambda_{3}}(\theta)=A+2 B \sum_{i=1}^{v} \cos \left(\Lambda_{3}-\theta_{i}\right)$, i.e., $\sigma\left(\tilde{H}_{\Lambda_{3}}^{3}\right)=\sigma_{\text {cont }}\left(\tilde{H}_{\Lambda_{3}}^{3}\right)=[A-2 B v, A+2 B v]$.

Now, using the obtained results and representation (8), we describe the structure of essential spectrum and the discrete spectrum of the operator ${ }^{3} \tilde{H}_{1 / 2}^{d}$ of third five-electron doublet state:

Theorem 4. At $v=1$ and $U<0$ the essential spectrum of the system of third five-electron doublet state operator ${ }^{3} \tilde{H}_{1 / 2}^{d}$ is exactly the union of four segments.

$$
\begin{aligned}
\sigma_{e s s}\left({ }^{3} \tilde{H}_{1 / 2}^{d}\right)= & {[a+c+e, b+d+f] \cup\left[a+e+\tilde{z}_{2}, b+f+\tilde{z}_{2}\right] } \\
& \bigcup\left[c+e+z_{1}, d+f+z_{1}\right] \cup\left[e+z_{1}+\tilde{z}_{2}, f+z_{1}+\tilde{z}_{2}\right] .
\end{aligned}
$$

The discrete spectrum of the operator ${ }^{3} \tilde{H}_{1 / 2}^{d}$ is empty: $\sigma_{\text {disc }}\left({ }^{3} \tilde{H}_{3 / 2}^{q}\right)=\varnothing$.

Here and hereafter $a=2 A-4 B \cos \frac{\Lambda_{1}}{2}, b=2 A+4 B \cos \frac{\Lambda_{1}}{2}$,

$c=2 A-4 B \cos \frac{\Lambda_{2}}{2}, d=2 A+4 B \cos \frac{\Lambda_{2}}{2}, e=A-2 B, f=A+2 B$,

$z_{1}=2 A-2 \sqrt{U^{2}+4 B^{2} \cos ^{2} \frac{\Lambda_{1}}{2}}, \quad \tilde{z}_{2}=2 A+2 \sqrt{U^{2}+4 B^{2} \cos ^{2} \frac{\Lambda_{2}}{2}}$.

Theorem 5. At $v=1$ and $U>0$ the essential spectrum of the system of third five-electron doublet state operator ${ }^{3} \tilde{H}_{1 / 2}^{d}$ is exactly the union of four segments.

$$
\begin{aligned}
\sigma_{e s s}\left({ }^{3} \tilde{H}_{1 / 2}^{d}\right)= & {[a+c+e, b+d+f] \cup\left[a+e+z_{2}, b+f+z_{2}\right] } \\
& \bigcup\left[c+e+\tilde{z}_{1}, d+f+\tilde{z}_{1}\right] \cup\left[e+\tilde{z}_{1}+z_{2}, f+\tilde{z}_{1}+z_{2}\right] .
\end{aligned}
$$

The discrete spectrum of the operator ${ }^{3} \tilde{H}_{1 / 2}^{d}$ is empty: $\sigma_{\text {disc }}\left({ }^{3} \tilde{H}_{3 / 2}^{q}\right)=\varnothing$.

Here $\tilde{z}_{1}=2 A+2 \sqrt{U^{2}+4 B^{2} \cos ^{2} \frac{\Lambda_{1}}{2}}, \quad z_{2}=2 A-2 \sqrt{U^{2}+4 B^{2} \cos ^{2} \frac{\Lambda_{2}}{2}}$. 
Proof. It follows from representation (8) that $\sigma\left({ }^{3} \tilde{H}_{1 / 2}^{d}\right)=\left\{\lambda+\mu+\theta: \lambda \in \sigma\left(\tilde{H}_{2 \Lambda_{1}}^{1}\right), \mu \in \sigma\left(\tilde{H}_{2 \Lambda_{2}}^{2}\right), \theta \in \sigma\left(\tilde{H}_{\Lambda_{3}}^{3}\right)\right\}$, and in the one-dimensional case, the continuous spectrum of operator $\tilde{H}_{2 \Lambda_{1}}^{1}$ is $\sigma_{\text {cont }}\left(\tilde{H}_{2 \Lambda_{1}}^{1}\right)=\left[2 A-4 B \cos \frac{\Lambda_{1}}{2}, 2 A+4 B \cos \frac{\Lambda_{1}}{2}\right]$, and at $U<0$, the discrete spectrum of $\tilde{H}_{2 \Lambda_{1}}^{1}$ consists of a single point $z_{1}$. The continuous spectrum of operator $\tilde{H}_{2 \Lambda_{2}}^{2}$ is $\sigma_{\text {cont }}\left(\tilde{H}_{2 \Lambda_{2}}^{2}\right)=\left[2 A-4 B \cos \frac{\Lambda_{2}}{2}, 2 A+4 B \cos \frac{\Lambda_{2}}{2}\right]$, and at $U<0$, the discrete spectrum of $\tilde{H}_{2 \Lambda_{2}}^{2}$ consists of a single point $\tilde{z}_{2}$. The spectra of the operator $\tilde{H}_{\Lambda_{3}}^{3}$ is a purely continuous and consists of the segment $[A-2 B, A+2 B]$. Therefore, the essential spectrum of the system of third doublet-state operator ${ }^{3} \tilde{H}_{1 / 2}^{d}$ is the union of four segments, and the third doublet-state operator ${ }^{3} \tilde{H}_{1 / 2}^{d}$ has no eigenvalues.

The Theorem 5 is proved totally similarly to Theorem 4 .

In the two-dimensional case to occur the analogous results.

We now consider the three-dimensional case.

Theorem 6. The following statements hold:

1) Let $v=3$ and $U<0, U<-\frac{2 B}{m}, M>m$, or $U<0, U<-\frac{2 B}{M}$, $M<m$. Then the essential spectrum of the system third five-electron doublet state operator ${ }^{3} \tilde{H}_{1 / 2}^{d}$ is the union of four segments.

$$
\begin{aligned}
\sigma_{\text {ess }}\left({ }^{3} \tilde{H}_{3 / 2}^{q}\right)= & {[a+c+e, b+d+f] \cup\left[a+e+\tilde{z}_{2}, b+f+\tilde{z}_{2}\right] } \\
& \bigcup\left[c+e+z_{1}, d+f+z_{1}\right] \cup\left[e+z_{1}+\tilde{z}_{2}, f+z_{1}+\tilde{z}_{2}\right] .
\end{aligned}
$$

The discrete spectrum of the operator ${ }^{3} \tilde{H}_{1 / 2}^{d}$ is empty: $\sigma_{\text {disc }}\left({ }^{3} \tilde{H}_{1 / 2}^{d}\right)=\varnothing$.

Here, $a=2 A-4 B \sum_{i=1}^{3} \cos \frac{\Lambda_{1}^{i}}{2}, \quad b=2 A+4 B \sum_{i=1}^{3} \cos \frac{\Lambda_{1}^{i}}{2}$,

$c=2 A-4 B \sum_{i=1}^{3} \cos \frac{\Lambda_{2}^{i}}{2}, \quad d=2 A+4 B \sum_{i=1}^{3} \cos \frac{\Lambda_{2}^{i}}{2}, \quad e=A-6 B, \quad f=A+6 B$ and $z_{1}, \tilde{z}_{2}$ are the eigenvalues of the operators $\tilde{H}_{2 \Lambda_{1}}^{1}, \tilde{H}_{2 \Lambda_{2}}^{2}$, correspondingly.

${ }_{2 B}^{2)}$ Let $v=3$ and $U<0,-\frac{2 B}{m} \leq U<-\frac{2 B}{M}$, and $M>m$ or $U<0$, $-\frac{2 B}{M} \leq U<-\frac{2 B}{m}$, and $M<m$. Then the essential spectrum of the system third five-electron doublet state operator ${ }^{3} \tilde{H}_{1 / 2}^{d}$ is the union of two segments. $\sigma_{e s s}\left({ }^{3} \tilde{H}_{1 / 2}^{d}\right)=[a+c+e, b+d+f] \bigcup\left[a+e+\tilde{z}_{2}, b+f+\tilde{z}_{2}\right]$ or

$\sigma_{\text {ess }}\left({ }^{3} \tilde{H}_{1 / 2}^{d}\right)=[a+c+e, b+d+f] \cup\left[c+e+z_{1}, d+f+z_{1}\right]$. The discrete spectrum of the operator ${ }^{3} \tilde{H}_{1 / 2}^{d}$ is empty: $\sigma_{\text {disc }}\left({ }^{3} \tilde{H}_{1 / 2}^{d}\right)=\varnothing$.

3) Let $v=3$ and $U<0,-\frac{2 B}{m} \leq U<0$, and $M<m$, or $U<0$, $-\frac{2 B}{M} \leq U<0$, and $M>m$. Then the essential spectrum of the system third five-electron doublet state operator ${ }^{3} \tilde{H}_{1 / 2}^{d}$ is consists of single segment. $\sigma_{\text {ess }}\left({ }^{3} \tilde{H}_{1 / 2}^{d}\right)=[a+c+e, b+d+f]$, and the discrete spectrum of the system third doublet-state operator ${ }^{3} \tilde{H}_{1 / 2}^{d}$ is empty: $\quad \sigma_{\text {disc }}\left({ }^{3} \tilde{H}_{1 / 2}^{d}\right)=\varnothing$.

Theorem 7. The following statements hold:

1) Let $v=3$ and $U>0, U>\frac{2 B}{m}, M>m$, or $U>0, U>\frac{2 B}{M}, M<m$. 
Then the essential spectrum of the system third five-electron doublet state operator ${ }^{3} \tilde{H}_{1 / 2}^{d}$ is the union of four segments.

$$
\begin{aligned}
\sigma_{e s s}\left({ }^{3} \tilde{H}_{3 / 2}^{q}\right)= & {[a+c+e, b+d+f] \cup\left[a+e+z_{2}, b+f+z_{2}\right] } \\
& \bigcup\left[c+e+\tilde{z}_{1}, d+f+\tilde{z}_{1}\right] \cup\left[e+\tilde{z}_{1}+z_{2}, f+\tilde{z}_{1}+z_{2}\right] .
\end{aligned}
$$

The discrete spectrum of the operator ${ }^{3} \tilde{H}_{1 / 2}^{d}$ is empty. $\sigma_{\text {disc }}\left({ }^{3} \tilde{H}_{1 / 2}^{d}\right)=\varnothing$.

Here, $a=2 A-4 B \sum_{i=1}^{3} \cos \frac{\Lambda_{1}^{i}}{2}, b=2 A+4 B \sum_{i=1}^{3} \cos \frac{\Lambda_{1}^{i}}{2}$,

$c=2 A-4 B \sum_{i=1}^{3} \cos \frac{\Lambda_{2}^{i}}{2}, d=2 A+4 B \sum_{i=1}^{3} \cos \frac{\Lambda_{2}^{i}}{2}, e=A-6 B, f=A+6 B$,

and $\tilde{z}_{1}$ is an eigenvalue of the operator $\tilde{H}_{2 \Lambda_{1}}^{1}$, and $z_{2}$ is an eigenvalue of the operator $\tilde{H}_{2 \Lambda_{2}}^{2}$.

2) Let $v=3$ and $U>0, \frac{2 B}{m} \leq U<\frac{2 B}{M}$, and $M<m$, or $U>0$, $\frac{2 B}{M} \leq U<\frac{2 B}{m}$, and $M>m$. Then the essential spectrum of the system third five-electron doublet state operator ${ }^{3} \tilde{H}_{1 / 2}^{d}$ is the union of two segments. $\sigma_{e s s}\left({ }^{3} \tilde{H}_{1 / 2}^{d}\right)=[a+c+e, b+d+f] \cup\left[a+e+z_{2}, b+f+z_{2}\right]$ or $\sigma_{e s s}\left({ }^{3} \tilde{H}_{1 / 2}^{d}\right)=[a+c+e, b+d+f] \cup\left[c+e+\tilde{z}_{1}, d+f+\tilde{z}_{1}\right]$, and the discrete spectrum of the system third doublet-state operator ${ }^{3} \tilde{H}_{1 / 2}^{d}$ is empty. $\sigma_{\text {disc }}\left({ }^{3} \tilde{H}_{1 / 2}^{d}\right)=\varnothing$.

3) Let $v=3$ and $U>0,0<U \leq \frac{2 B}{m}$, and $M<m$, or $U>0$, $0<U \leq \frac{2 B}{M}$, and $M>m$. Then the essential spectrum of the system third five-electron doublet state operator ${ }^{3} \tilde{H}_{1 / 2}^{d}$ is consists of single segment. $\sigma_{\text {ess }}\left({ }^{3} \tilde{H}_{1 / 2}^{d}\right)=[a+c+e, b+d+f]$, and the discrete spectrum of the system third five-electron doublet state operator ${ }^{3} \tilde{H}_{1 / 2}^{d}$ is empty. $\quad \sigma_{\text {disc }}\left({ }^{3} \tilde{H}_{1 / 2}^{d}\right)=\varnothing$.

We now consider the three-dimensional case. Let $v=3$, and $\Lambda_{1}=\left(\Lambda_{1}^{0}, \Lambda_{1}^{0}, \Lambda_{1}^{0}\right)$, and $\Lambda_{2}=\left(\Lambda_{2}^{0}, \Lambda_{2}^{0}, \Lambda_{2}^{0}\right)$.

Then the continuous spectrum of the operator $\tilde{H}_{2 \Lambda_{1}}^{1}$ is consists of the segment

$$
\sigma_{\text {cont }}\left(\tilde{H}_{2 \Lambda_{1}}^{1}\right)=G_{\Lambda_{1}}^{3}=\left[2 A-12 B \cos \frac{\Lambda_{1}^{0}}{2}, 2 A+12 B \cos \frac{\Lambda_{1}^{0}}{2}\right] .
$$

We consider the Watson integral $W=\frac{1}{\pi^{3}} \int_{0}^{\pi} \int_{0}^{\pi} \int_{0}^{\pi} \frac{3 \mathrm{~d} x \mathrm{~d} y \mathrm{~d} z}{3-\cos x-\cos y-\cos z} \approx 1.516$. (see. [20]). Because the measure $v$ is normalized, $\int_{T^{3}} \frac{\mathrm{d} s_{1} \mathrm{~d} s_{2} \mathrm{~d} s_{3}}{3-\sum_{i=1}^{3} \cos \left(\Lambda_{3}^{i}-s_{i}\right)}=\frac{W}{3}$.

Theorem 8. At $v=3$ and $U<0$ and the total quasimomentum $\Lambda_{1}$ of the system have the form $\Lambda_{1}=\left(\Lambda_{1}^{1}, \Lambda_{1}^{2}, \Lambda_{1}^{3}\right)=\left(\Lambda_{1}^{0}, \Lambda_{1}^{0}, \Lambda_{1}^{0}\right)$. Then the operator $\tilde{H}_{2 \Lambda_{1}}^{1}$ has a unique eigenvalue $z_{1}$ if $U<-\frac{6 B \cos \frac{\Lambda_{1}^{0}}{2}}{W}$, that is below of continuous spectrum of operator $\tilde{H}_{2 \Lambda_{1}}^{1}$. Otherwise, the operator $\tilde{H}_{2 \Lambda_{1}}^{1}$ has no eigenvalue, that is below of continuous spectrum of operator $\tilde{H}_{2 \Lambda_{1}}^{1}$.

Theorem 9. At $v=3$ and $U>0$ and the total quasimomentum $\Lambda_{1}$ of the system have the form $\Lambda_{1}=\left(\Lambda_{1}^{1}, \Lambda_{1}^{2}, \Lambda_{1}^{3}\right)=\left(\Lambda_{1}^{0}, \Lambda_{1}^{0}, \Lambda_{1}^{0}\right)$. Then the operator 
$\tilde{H}_{2 \Lambda_{1}}^{1}$ has a unique eigenvalue $\tilde{z}_{1}$ if $U>\frac{6 B \cos \frac{\Lambda_{1}^{0}}{2}}{W}$, that is above of continuous spectrum of operator $\tilde{H}_{2 \Lambda_{1}}^{1}$. Otherwise, the operator $\tilde{H}_{2 \Lambda_{1}}^{1}$ has no eigenvalue, that is above of continuous spectrum of operator $\tilde{H}_{2 \Lambda_{1}}^{1}$.

In this case the continuous spectrum of the operator $\tilde{H}_{2 \Lambda_{2}}^{2}$ is consists of the segment

$$
\sigma_{\text {cont }}\left(\tilde{H}_{2 \Lambda_{2}}^{2}\right)=G_{\Lambda_{2}}^{3}=\left[2 A-12 B \cos \frac{\Lambda_{2}^{0}}{2}, 2 A+12 B \cos \frac{\Lambda_{2}^{0}}{2}\right] .
$$

Theorem 10. At $v=3$ and $U<0$ and the total quasimomentum $\Lambda_{2}$ of the system have the form $\Lambda_{2}=\left(\Lambda_{2}^{1}, \Lambda_{2}^{2}, \Lambda_{2}^{3}\right)=\left(\Lambda_{2}^{0}, \Lambda_{2}^{0}, \Lambda_{2}^{0}\right)$. Then the operator $\tilde{H}_{2 \Lambda_{2}}^{2}$ has a unique eigenvalue $\tilde{z}_{2}$ if $U<-\frac{6 B \cos \frac{\Lambda_{2}^{0}}{2}}{W}$, that is above of continuous spectrum of operator $\tilde{H}_{2 \Lambda_{2}}^{2}$. Otherwise, the operator $\tilde{H}_{2 \Lambda_{2}}^{2}$ has no eigenvalue, that is above of continuous spectrum of operator $\tilde{H}_{2 \Lambda_{2}}^{2}$.

Theorem 11. At $v=3$ and $U>0$ and the total quasimomentum $\Lambda_{2}$ of the system have the form $\Lambda_{2}=\left(\Lambda_{2}^{1}, \Lambda_{2}^{2}, \Lambda_{2}^{3}\right)=\left(\Lambda_{2}^{0}, \Lambda_{2}^{0}, \Lambda_{2}^{0}\right)$. Then the operator $\tilde{H}_{2 \Lambda_{2}}^{2}$ has a unique eigenvalue $z_{2}$ if $U>\frac{6 B \cos \frac{\Lambda_{2}^{0}}{2}}{W}$, that is below of continuous spectrum of operator $\tilde{H}_{2 \Lambda_{2}}^{2}$. Otherwise, the operator $\tilde{H}_{2 \Lambda_{2}}^{2}$ has no eigenvalue, that is below of continuous spectrum of operator $\tilde{H}_{2 \Lambda_{2}}^{2}$.

We now using the obtaining results and representation (8), we can describe the structure of essential spectrum and discrete spectrum of the operator of third five-electron quartet state:

Let $v=3$ and $\Lambda_{1}=\left(\Lambda_{1}^{0}, \Lambda_{1}^{0}, \Lambda_{1}^{0}\right)$, and $\Lambda_{2}=\left(\Lambda_{2}^{0}, \Lambda_{2}^{0}, \Lambda_{2}^{0}\right)$.

Theorem 12. The following statements hold:

1) Let $U<0$, and $U<-\frac{6 B \cos \frac{\Lambda_{1}^{0}}{2}}{W}, \cos \frac{\Lambda_{1}^{0}}{2}>\cos \frac{\Lambda_{2}^{0}}{2}$, or $U<0$,

$U<-\frac{6 B \cos \frac{\Lambda_{2}^{0}}{2}}{W}, \cos \frac{\Lambda_{1}^{0}}{2}<\cos \frac{\Lambda_{2}^{0}}{2}$. Then the essential spectrum of the system third five-electron doublet state operator ${ }^{3} \tilde{H}_{1 / 2}^{d}$ is the union of four segments. $\begin{aligned} \sigma_{e s s}\left({ }^{3} \tilde{H}_{1 / 2}^{d}\right)= & {\left[a_{1}+c_{1}+e_{1}, b_{1}+d_{1}+f_{1}\right] \cup\left[a_{1}+e_{1}+\tilde{z}_{2}, b_{1}+f_{1}+\tilde{z}_{2}\right] } \\ & \bigcup\left[c_{1}+e_{1}+z_{1}, d_{1}+f_{1}+z_{1}\right] \cup\left[e_{1}+z_{1}+\tilde{z}_{2}, f_{1}+z_{1}+\tilde{z}_{2}\right] . \text { The discrete }\end{aligned}$ spectrum of the operator ${ }^{3} \tilde{H}_{1 / 2}^{d}$ is empty: $\quad \sigma_{\text {disc }}\left({ }^{3} \tilde{H}_{3 / 2}^{q}\right)=\varnothing$.

Here and hereafter $a_{1}=2 A-12 B \cos \frac{\Lambda_{1}^{0}}{2}, b_{1}=2 A+12 B \cos \frac{\Lambda_{1}^{0}}{2}$, $c_{1}=2 A-12 B \cos \frac{\Lambda_{2}^{0}}{2}, \quad d_{1}=2 A+12 B \cos \frac{\Lambda_{2}^{0}}{2}, \quad e_{1}=A-6 B, f_{1}=A+6 B$, and $z_{1}$ is an eigenvalue of the operator $\tilde{H}_{2 \Lambda_{1}}^{1}$, and $\tilde{z}_{2}$ is an eigenvalue of the oper- 
ator $\tilde{H}_{2 \Lambda_{2}}^{2}$.

2) Let $U<0$, and $-\frac{6 B \cos \frac{\Lambda_{1}^{0}}{2}}{W} \leq U<-\frac{6 B \cos \frac{\Lambda_{2}^{0}}{2}}{W}, \cos \frac{\Lambda_{1}^{0}}{2}>\cos \frac{\Lambda_{2}^{0}}{2}$, or $-\frac{6 B \cos \frac{\Lambda_{2}^{0}}{2}}{W} \leq U<-\frac{6 B \cos \frac{\Lambda_{1}^{0}}{2}}{W}, \cos \frac{\Lambda_{1}^{0}}{2}<\cos \frac{\Lambda_{2}^{0}}{2}$. Then the essential spectrum of the system third five-electron doublet state operator ${ }^{3} \tilde{H}_{1 / 2}^{d}$ is the union of two segments: $\sigma_{\text {ess }}\left({ }^{3} \tilde{H}_{1 / 2}^{d}\right)=\left[a_{1}+c_{1}+e_{1}, b_{1}+d_{1}+f_{1}\right] \cup\left[a_{1}+e_{1}+\tilde{z}_{2}, b_{1}+f_{1}+\tilde{z}_{2}\right]$, or $\sigma_{\text {ess }}\left({ }^{3} \tilde{H}_{1 / 2}^{d}\right)=\left[a_{1}+c_{1}+e_{1}, b_{1}+d_{1}+f_{1}\right] \cup\left[c_{1}+e_{1}+z_{1}, d_{1}+f_{1}+z_{1}\right]$. The discrete spectrum of the operator ${ }^{3} \tilde{H}_{1 / 2}^{d}$ is empty: $\quad \sigma_{\text {disc }}\left({ }^{3} \tilde{H}_{1 / 2}^{d}\right)=\varnothing$.

3) Let $U<0,-\frac{6 B \cos \frac{\Lambda_{1}^{0}}{2}}{W} \leq U<0, \cos \frac{\Lambda_{1}^{0}}{2}<\cos \frac{\Lambda_{2}^{0}}{2}$ or

$-\frac{6 B \cos \frac{\Lambda_{2}^{0}}{2}}{W} \leq U<0, \cos \frac{\Lambda_{1}^{0}}{2}>\cos \frac{\Lambda_{2}^{0}}{2}$. Then the essential spectrum of the system third five-electron doublet state operator ${ }^{3} \tilde{H}_{1 / 2}^{d}$ is consists of single segment. $\sigma_{\text {ess }}\left({ }^{3} \tilde{H}_{1 / 2}^{d}\right)=\left[a_{1}+c_{1}+e_{1}, b_{1}+d_{1}+f_{1}\right]$, and discrete spectrum of the system third five-electron doublet state operator ${ }^{3} \tilde{H}_{1 / 2}^{d}$ is empty. $\sigma_{\text {disc }}\left({ }^{3} \tilde{H}_{1 / 2}^{d}\right)=\varnothing$.

Theorem 13. The following statements hold:

1) Let $U>0$, and $U>\frac{6 B \cos \frac{\Lambda_{1}^{0}}{2}}{W}, \cos \frac{\Lambda_{1}^{0}}{2}>\cos \frac{\Lambda_{2}^{0}}{2}$, or $U>0$, $U>\frac{6 B \cos \frac{\Lambda_{2}^{0}}{2}}{W}, \cos \frac{\Lambda_{1}^{0}}{2}<\cos \frac{\Lambda_{2}^{0}}{2}$. Then the essential spectrum of the system third five-electron doublet state operator ${ }^{3} \tilde{H}_{1 / 2}^{d}$ is the union of four segments. $\begin{aligned} \sigma_{\text {ess }}\left({ }^{3} \tilde{H}_{1 / 2}^{d}\right)= & {\left[a_{1}+c_{1}+e_{1}, b_{1}+d_{1}+f_{1}\right] \cup\left[a_{1}+e_{1}+z_{2}, b_{1}+f_{1}+z_{2}\right] . \text { The discrete } } \\ & \cup\left[c_{1}+e_{1}+\tilde{z}_{1}, d_{1}+f_{1}+\tilde{z}_{1}\right] \cup\left[e_{1}+\tilde{z}_{1}+z_{2}, f_{1}+\tilde{z}_{1}+z_{2}\right]\end{aligned}$ spectrum of the operator ${ }^{3} \tilde{H}_{1 / 2}^{d}$ is empty: $\quad \sigma_{\text {disc }}\left({ }^{3} \tilde{H}_{3 / 2}^{q}\right)=\varnothing$.

Here and hereafter $a_{1}=2 A-12 B \cos \frac{\Lambda_{1}^{0}}{2}, b_{1}=2 A+12 B \cos \frac{\Lambda_{1}^{0}}{2}$, $c_{1}=2 A-12 B \cos \frac{\Lambda_{2}^{0}}{2}, \quad d_{1}=2 A+12 B \cos \frac{\Lambda_{2}^{0}}{2}, e_{1}=A-6 B, f_{1}=A+6 B$, and $\tilde{z}_{1}$ is an eigenvalue of the operator $\tilde{H}_{2 \Lambda_{1}}^{1}$, and $z_{2}$ is an eigenvalue of the operator $\tilde{H}_{2 \Lambda_{2}}^{2}$.

2) Let $U>0$, and $\frac{6 B \cos \frac{\Lambda_{1}^{0}}{2}}{W} \leq U<\frac{6 B \cos \frac{\Lambda_{2}^{0}}{2}}{W}, \cos \frac{\Lambda_{1}^{0}}{2}<\cos \frac{\Lambda_{2}^{0}}{2}$, or $\frac{6 B \cos \frac{\Lambda_{2}^{0}}{2}}{W} \leq U<\frac{6 B \cos \frac{\Lambda_{1}^{0}}{2}}{W}, \cos \frac{\Lambda_{1}^{0}}{2}>\cos \frac{\Lambda_{2}^{0}}{2}$. Then the essential spectrum of the system third five-electron doublet state operator ${ }^{3} \tilde{H}_{1 / 2}^{d}$ is the union of two segments. $\sigma_{\text {ess }}\left({ }^{3} \tilde{H}_{1 / 2}^{d}\right)=\left[a_{1}+c_{1}+e_{1}, b_{1}+d_{1}+f_{1}\right] \cup\left[c_{1}+e_{1}+\tilde{z}_{1}, d_{1}+f_{1}+\tilde{z}_{1}\right]$, or 
$\sigma_{\text {ess }}\left({ }^{3} \tilde{H}_{1 / 2}^{d}\right)=\left[a_{1}+c_{1}+e_{1}, b_{1}+d_{1}+f_{1}\right] \cup\left[a_{1}+e_{1}+z_{2}, b_{1}+f_{1}+z_{2}\right]$. The discrete spectrum of the operator ${ }^{3} \tilde{H}_{1 / 2}^{d}$ is empty. $\quad \sigma_{\text {disc }}\left({ }^{3} \tilde{H}_{1 / 2}^{d}\right)=\varnothing$.

3) Let $U>0$, and $0<U \leq \frac{6 B \cos \frac{\Lambda_{1}^{0}}{2}}{W}, \cos \frac{\Lambda_{1}^{0}}{2}<\cos \frac{\Lambda_{2}^{0}}{2}$ or

$0<U \leq \frac{6 B \cos \frac{\Lambda_{2}^{0}}{2}}{W}, \cos \frac{\Lambda_{1}^{0}}{2}>\cos \frac{\Lambda_{2}^{0}}{2}$. Then the essential spectrum of the system third five-electron doublet state operator ${ }^{3} \tilde{H}_{1 / 2}^{d}$ is consists of single segment: $\sigma_{\text {ess }}\left({ }^{3} \tilde{H}_{1 / 2}^{d}\right)=\left[a_{1}+c_{1}+e_{1}, b_{1}+d_{1}+f_{1}\right]$ and the discrete spectrum of the system third five-electron doublet state operator ${ }^{3} \tilde{H}_{1 / 2}^{d}$ is empty. $\sigma_{\text {disc }}\left({ }^{3} \tilde{H}_{1 / 2}^{d}\right)=\varnothing$.

Consequently, the essential spectrum of the system third five-electron doublet state operator ${ }^{3} \tilde{H}_{1 / 2}^{d}$ is the union of no more than four segments, and discrete spectrum of the operator ${ }^{3} \tilde{H}_{1 / 2}^{d}$ is empty.

\section{Fourth Doublet State}

The fourth doublet state corresponds to the basic functions ${ }^{4} d_{m, n, r, t, l \in Z^{v}}^{1 / 2}=a_{m, \downarrow}^{+} a_{n, \uparrow}^{+} a_{r, \uparrow}^{+} a_{t, \uparrow}^{+} a_{l, \downarrow}^{+} \varphi_{0}$. The subspace ${ }^{4} \mathcal{H}_{1 / 2}^{d}$, corresponding to the fourth five-electron doublet state is the set of all vectors of the form

$$
{ }^{4} \psi_{1 / 2}^{d}=\sum_{m, n, r, t, l \in Z^{v}} f(m, n, r, t, l)^{4} d_{m, n, r, t, l \in Z^{v}}^{1 / 2}, f \in l_{2}^{a s},
$$

where $l_{2}^{a s}$ is the subspace of antisymmetric functions in the space $l_{2}\left(\left(Z^{v}\right)^{5}\right)$.

The restriction ${ }^{4} H_{1 / 2}^{d}$ of $H$ to the subspace ${ }^{4} \mathcal{H}_{1 / 2}^{d}$, is called the five-electron fourth doublet state operator.

Theorem 14. The subspace ${ }^{4} \mathcal{H}_{1 / 2}^{d}$ is invariant under the operator $H$, and the operator ${ }^{4} H_{1 / 2}^{d}$ is a bounded self-adjoint operator. It generates a bounded self-adjoint operator ${ }^{4} \bar{H}_{1 / 2}^{d}$ acting in the space $l_{2}^{a s}$ as

$$
\begin{aligned}
& \left({ }^{4} \bar{H}_{1 / 2}^{d} f\right)(m, n, r, t, l) \\
& =5 A f(m, n, r, t, l)+B \sum_{\tau}[f(m+\tau, n, r, t, l)+f(m, n+\tau, r, t, l) \\
& +f(m, n, r+\tau, t, l)+f(m, n, r, t+\tau, l)+f(m, n, r, t, l+\tau)] \\
& +U\left[\delta_{m, n}+\delta_{m, r}+\delta_{m, t}+\delta_{n, l}+\delta_{t, l}+\delta_{r, l}\right] f(m, n, r, t, l),
\end{aligned}
$$

where $\delta_{k, j}$ is the Kronecker symbol. The operator ${ }^{4} H_{1 / 2}^{d}$ acts on a vector ${ }^{4} \psi_{1 / 2}^{d} \in{ }^{4} \mathcal{H}_{1 / 2}^{d}$ as

$$
{ }^{4} H_{1 / 2}^{d}{ }^{4} \psi_{1 / 2}^{d}=\sum_{m, n, r, t, l \in Z^{v}}\left({ }^{4} \bar{H}_{1 / 2}^{d} f\right)(m, n, r, t, l)^{4} d_{m, n, r, t, l \in Z^{v}}^{1 / 2} .
$$

Proof. The proof of the theorem can be obtained from the explicit form of the action of $H$ on vectors $\psi \in{ }^{4} \mathcal{H}_{1 / 2}^{d}$ using the standard anticommutation relations between electron creation and annihilation operators at lattice sites, $\left\{a_{m, \gamma}, a_{n, \beta}^{+}\right\}=\delta_{m, n} \delta_{\gamma, \beta},\left\{a_{m, \gamma}, a_{n, \beta}\right\}=\left\{a_{m, \gamma}^{+}, a_{n, \beta}^{+}\right\}=\theta$, and also take into account that $a_{m, \gamma} \varphi_{0}=\theta$, where $\theta$ is the zero element of ${ }^{4} \mathcal{H}_{1 / 2}^{d}$. This yields the state- 
ment of the theorem.

We set ${ }^{4} \tilde{H}_{1 / 2}^{d}=\mathcal{F}^{4} \bar{H}_{1 / 2}^{d} \mathcal{F}^{-1}$. In the quasimomentum representation, the operator ${ }^{4} \tilde{H}_{1 / 2}^{d}$ acts in the Hilbert space $L_{2}^{a s}\left(\left(T^{v}\right)^{5}\right)$ as

$$
\begin{aligned}
& { }^{4} \tilde{H}_{1 / 2}^{d}{ }^{4} \psi_{1 / 2}^{d} \\
& =\left\{5 A+2 B \sum_{i=1}^{v}\left[\cos \lambda_{i}+\cos \mu_{i}+\cos \gamma_{i}+\cos \theta_{i}+\cos \eta_{i}\right]\right\} f(\lambda, \mu, \gamma, \theta, \eta) \\
& +U \int_{T^{v}}[f(s, \lambda+\mu-s, \gamma, \theta, \eta)+f(s, \mu, \lambda+\gamma-s, \theta, \eta) \\
& +f(s, \mu, \gamma, \lambda+\theta-s, \eta)+f(\lambda, s, \gamma, \theta, \mu+\eta-s) \\
& +f(\lambda, \mu, s, \theta, \gamma+\eta-s)+f(\lambda, \mu, \gamma, s, \theta+\eta-s)] \mathrm{d} s,
\end{aligned}
$$

where $L_{2}^{a s}\left(\left(T^{v}\right)^{5}\right)$ is the subspace of antisymmetric functions in $L_{2}\left(\left(T^{v}\right)^{5}\right)$.

Taking into account that the function $f(\lambda, \mu, \gamma, \theta, \eta)$ is antisymmetric, we can rewrite Formula (13) as

$$
{ }^{4} \tilde{H}_{1 / 2}^{d}=\tilde{H}_{2}^{4} \otimes I \otimes I+I \otimes \tilde{H}_{2}^{5} \otimes I+I \otimes I \otimes \tilde{H}_{2}^{6},
$$

where

$$
\begin{aligned}
\left(\tilde{H}_{2}^{4} f\right)(\lambda, \mu)= & \left\{2 A+2 B \sum_{i=1}^{v}\left[\cos \lambda_{i}+\cos \mu_{i}\right]\right\} f(\lambda, \mu) \\
& +U \int_{T^{v}} f(s, \lambda+\mu-s) \mathrm{d} s+U \int_{T^{v}} f(s, \lambda+\theta-s) \mathrm{d} s, \\
\left(\tilde{H}_{2}^{5} f\right)(\gamma, \theta)= & \left\{2 A+2 B \sum_{i=1}^{v}\left[\cos \gamma_{i}+\cos \theta_{i}\right]\right\} f(\gamma, \theta) \\
& -U \int_{T^{v}} f(s, \lambda+\gamma-s) \mathrm{d} s-U \int_{T^{v}} f(s, \gamma+\eta-s) \mathrm{d} s, \\
\left(\tilde{H}_{2}^{6} f\right)(\theta, \eta)=\left\{A+2 B \sum_{i=1}^{v} \cos \eta_{i}\right\} f(\theta, \eta)+U \int_{T^{v}} f(s, \mu+\eta-s) \mathrm{d} s & +U \int_{T^{v}} f(s, \theta+\eta-s) \mathrm{d} s,
\end{aligned}
$$

and $I$ is the unit operator.

Taking into account that $\lambda, \mu, \gamma, \theta, \eta \in T^{v}$, we can express the action of operators $\tilde{H}_{2}^{4}, \tilde{H}_{2}^{5}, \tilde{H}_{2}^{6}$ in the form

$$
\begin{aligned}
\left(\tilde{H}_{2}^{4} f\right)(\lambda, \mu)=\left\{2 A+2 B \sum_{i=1}^{v}\left[\cos \lambda_{i}+\cos \mu_{i}\right]\right\} f(\lambda, \mu) \\
+2 U \int_{T^{v}} f(s, \lambda+\mu-s) \mathrm{d} s, \\
\left(\tilde{H}_{2}^{5} f\right)(\gamma, \theta)=\left\{2 A+2 B \sum_{i=1}^{v}\left[\cos \gamma_{i}+\cos \theta_{i}\right]\right\} f(\gamma, \theta) \\
-2 U \int_{T^{v}} f(s, \gamma+\theta-s) \mathrm{d} s, \\
\left(\tilde{H}_{2}^{6} f\right)(\theta, \eta)=\left\{A+2 B \sum_{i=1}^{v} \cos \eta_{i}\right\} f(\theta, \eta)+2 U \int_{T^{v}} f(s, \theta+\eta-s) \mathrm{d} s .
\end{aligned}
$$

We must therefore investigate the spectrum and bound states (antibound states) of the operators $\tilde{H}_{2}^{4}, \tilde{H}_{2}^{5}$ and $\tilde{H}_{2}^{6}$. Let the total quasimomentum of the two-electron systems by fixed: $\Lambda_{1}=\lambda+\mu, \Lambda_{2}=\gamma+\theta, \Lambda_{3}=\theta+\eta$. We let 
$L_{2}\left(\Gamma_{\Lambda_{1}}\right)$ (correspondingly, $L_{2}\left(\Gamma_{\Lambda_{2}}\right)$ and $\left.L_{2}\left(\Gamma_{\Lambda_{3}}\right)\right)$ denote the space of functions that are square integrable on the manifold $\Gamma_{\Lambda_{1}}=\left\{(\lambda, \mu): \lambda+\mu=\Lambda_{1}\right\}$ (correspondingly, $\Gamma_{\Lambda_{2}}=\left\{(\gamma, \theta): \gamma+\theta=\Lambda_{2}\right\}$ and $\Gamma_{\Lambda_{3}}=\left\{(\theta, \eta): \theta+\eta=\Lambda_{3}\right\}$ ). That the operator $\tilde{H}_{2}^{4}, \tilde{H}_{2}^{5}$ and $\tilde{H}_{2}^{6}$ and the space $\tilde{\mathcal{H}}_{2} \equiv L_{2}\left(\left(T^{v}\right)^{2}\right)$ can be decomposed into a direct integral $\tilde{H}_{2}^{4}=\bigoplus \int_{T^{v}} \tilde{H}_{2 \Lambda_{1}}^{4} \mathrm{~d} \Lambda_{1}$ (correspondingly, $\tilde{H}_{2}^{5}=\bigoplus \int_{T^{v}} \tilde{H}_{2 \Lambda_{2}}^{5} \mathrm{~d} \Lambda_{2} \quad$ and $\left.\tilde{H}_{2}^{6}=\bigoplus \int_{T^{v}} \tilde{H}_{2 \Lambda_{3}}^{6} \mathrm{~d} \Lambda_{3}\right), \quad \tilde{\mathcal{H}}_{2}=\bigoplus \int_{T^{v}} \tilde{\mathcal{H}}_{2 \Lambda_{1}} \mathrm{~d} \Lambda_{1} \quad$ (correspondingly, $\quad \tilde{\mathcal{H}}_{2}=\bigoplus \int_{T^{v}} \tilde{\mathcal{H}}_{2 \Lambda_{2}} \mathrm{~d} \Lambda_{2} \quad$ and $\left.\tilde{\mathcal{H}}_{2}=\bigoplus \int_{T^{v}} \tilde{\mathcal{H}}_{2 \Lambda_{3}} \mathrm{~d} \Lambda_{3}\right)$. Each operator $\tilde{H}_{2 \Lambda_{1}}^{4}$ (correspondingly, $\tilde{H}_{2 \Lambda_{2}}^{5}$ and $\tilde{H}_{2 \Lambda_{3}}^{6}$ ) acts in $\tilde{\mathcal{H}}_{2 \Lambda_{1}}$ (correspondingly, $\tilde{\mathcal{H}}_{2 \Lambda_{2}}$ and $\tilde{\mathcal{H}}_{2 \Lambda_{3}}$ ) as

$$
\left(\tilde{H}_{2 \Lambda_{1}}^{4} f_{\Lambda_{1}}\right)(\lambda)=\left\{2 A+4 B \sum_{i=1}^{v} \cos \frac{\Lambda_{1}^{i}}{2} \cos \left(\frac{\Lambda_{1}^{i}}{2}-\lambda_{i}\right)\right\} f_{\Lambda_{1}}(\lambda)+2 U \int_{T^{v}} f_{\Lambda_{1}}(s) \mathrm{d} s,
$$

correspondingly,

$$
\begin{gathered}
\left(\tilde{H}_{2 \Lambda_{2}}^{5} f_{\Lambda_{2}}\right)(\gamma)=\left\{2 A+4 B \sum_{i=1}^{v} \cos \frac{\Lambda_{2}^{i}}{2} \cos \left(\frac{\Lambda_{2}^{i}}{2}-\gamma_{i}\right)\right\} f_{\Lambda_{2}}(\gamma)-2 U \int_{T^{v}} f_{\Lambda_{2}}(s) \mathrm{d} s \\
\left(\tilde{H}_{2 \Lambda_{3}}^{6} f_{\Lambda_{3}}\right)(\theta)=\left\{A+2 B \sum_{i=1}^{v} \cos \left(\Lambda_{3}^{i}-\theta_{i}\right)\right\} f_{\Lambda_{3}}(\theta)+2 U \int_{T^{v}} f_{\Lambda_{3}}(s) \mathrm{d} s
\end{gathered}
$$

where $f_{\Lambda_{1}}(\lambda)=f\left(\lambda, \Lambda_{1}-\lambda\right) \quad$ (correspondingly, $f_{\Lambda_{2}}(\gamma)=f\left(\gamma, \Lambda_{2}-\gamma\right)$ and $\left.f_{\Lambda_{3}}(\theta)=f\left(\theta, \Lambda_{3}-\theta\right)\right)$.

The continuous spectrum of operator $\tilde{H}_{2 \Lambda_{1}}^{4}$ (correspondingly, $\tilde{H}_{2 \Lambda_{2}}^{5}$ and $\tilde{H}_{2 \Lambda_{3}}^{6}$ ) does not depend on the parameter $U$ and consists of the intervals $G_{\Lambda_{1}}^{v}=\left[2 A-4 B \sum_{i=1}^{v} \cos \frac{\Lambda_{1}^{i}}{2}, 2 A+4 B \sum_{i=1}^{v} \cos \frac{\Lambda_{1}^{i}}{2}\right]$ (correspondingly,

$G_{\Lambda_{2}}^{v}=\left[2 A-4 B \sum_{i=1}^{v} \cos \frac{\Lambda_{2}^{i}}{2}, 2 A+4 B \sum_{i=1}^{v} \cos \frac{\Lambda_{2}^{i}}{2}\right]$ and

$\left.G_{\Lambda_{3}}^{v}=[A-2 B v, A+2 B v]\right)$.

$$
\text { We set } D_{\Lambda_{1}}^{v}(z)=1+2 U \int_{T^{v}} \frac{\mathrm{d} s_{1} \mathrm{~d} s_{2} \cdots \mathrm{d} s_{v}}{2 A+4 B \sum_{i=1}^{v} \cos \frac{\Lambda_{1}^{i}}{2} \cos \left(\frac{\Lambda_{1}^{i}}{2}-s_{i}\right)-z} \text {. }
$$

The analogue of the Lemma 2 holds for the in this case. We consider the one-dimensional case.

Theorem 15. 1) At $v=1$ and $U<0$ and for all values of the parameter of the Hamiltonian, the operator $\tilde{H}_{2 \Lambda_{1}}^{4}$ has a unique eigenvalue

$z_{1}=2 A-2 \sqrt{U^{2}+4 B^{2} \cos ^{2} \frac{\Lambda_{1}}{2}}$ that is below the continuous spectrum of $\tilde{H}_{2 \Lambda_{1}}^{4}$, i.e., $z_{1}<m_{\Lambda_{1}}^{1}$.

2) At $v=1$ and $U>0$ and for all values of the parameter of the Hamiltonian, the operator $\tilde{H}_{2 \Lambda_{1}}^{4}$ has a unique eigenvalue $\tilde{z}_{1}=2 A+2 \sqrt{U^{2}+4 B^{2} \cos ^{2} \frac{\Lambda_{1}}{2}}$ that is above the continuous spectrum of $\tilde{H}_{2 \Lambda_{1}}^{4}$. i.e., $\tilde{z}_{1}>M_{\Lambda_{1}}^{1}$. 
Proof. If $U<0$, then in the one-dimensional case, the function $D_{\Lambda_{1}}^{v}(z)$ is a monotone decreasing function on $\left(-\infty, m_{\Lambda_{1}}^{1}\right)$ and $\left(M_{\Lambda_{1}}^{1}, \infty\right)$, i.e., outside the continuous spectrum domain of the operator $\tilde{H}_{2 \Lambda_{1}}^{4}$. For $z<m_{\Lambda_{1}}^{1}$ the function $D_{\Lambda_{1}}^{v}(z)$ decreases from 1 to $-\infty, D_{\Lambda_{1}}^{v}(z) \rightarrow 1$ as $z \rightarrow-\infty, D_{\Lambda_{1}}^{v}(z) \rightarrow-\infty$ as $z \rightarrow m_{\Lambda_{1}}^{1}-0$. Therefore, below the value $m_{\Lambda_{1}}^{1}$, the function $D_{\Lambda_{1}}^{v}(z)$ has a single zero at the point $z=z_{1}=2 A-2 \sqrt{U^{2}+4 B^{2} \cos ^{2} \frac{\Lambda_{1}}{2}}$. For $z>M_{\Lambda_{1}}^{1}$, and $U<0$, the function $D_{\Lambda_{1}}^{v}(z)$ decreases from $+\infty$ to $1, D_{\Lambda_{1}}^{v}(z) \rightarrow+\infty$ as $z \rightarrow M_{\Lambda_{1}}^{1}+0, D_{\Lambda_{1}}^{v}(z) \rightarrow 1$ as $z \rightarrow+\infty$. Therefore, above the value $M_{\Lambda_{1}}^{1}$, the function $D_{\Lambda_{1}}^{v}(z)$ cannot vanish. If $U>0$, then the function $D_{\Lambda_{1}}^{v}(z)$ is a monotone increasing function on $\left(-\infty, m_{\Lambda_{1}}^{1}\right)$ and $\left(M_{\Lambda_{1}}^{1}, \infty\right)$, i.e., outside the continuous spectrum domain of the operator $\tilde{H}_{2 \Lambda_{1}}^{1}$. For $z<m_{\Lambda_{1}}^{1}$ the function $D_{\Lambda_{1}}^{v}(z)$ increases from 1 to $+\infty, D_{\Lambda_{1}}^{v}(z) \rightarrow 1$ as $z \rightarrow-\infty, D_{\Lambda_{1}}^{v}(z) \rightarrow+\infty$ as $z \rightarrow m_{\Lambda_{1}}^{1}-0$. Therefore, below the value $m_{\Lambda_{1}}^{1}$, the function $D_{\Lambda_{1}}^{v}(z)$ cannot vanish. For $z>M_{\Lambda_{1}}^{1}$, and $U>0$, the function $D_{\Lambda_{1}}^{v}(z)$ increases from $-\infty$ to $1, D_{\Lambda_{1}}^{v}(z) \rightarrow-\infty$ as $z \rightarrow M_{\Lambda_{1}}^{1}+0, D_{\Lambda_{1}}^{v}(z) \rightarrow 1$ as $z \rightarrow+\infty$. Therefore, above the value $M_{\Lambda_{1}}^{1}, D_{\Lambda_{1}}^{v}(z)$ has a single zero at the point $\tilde{z}_{1}=2 A+2 \sqrt{U^{2}+4 B^{2} \cos ^{2} \frac{\Lambda_{1}}{2}}$.

In the two-dimensional case, we have analogous results.

We consider three-dimensional case. Denote $m=\int_{T^{3}} \frac{\mathrm{d} s_{1} \mathrm{~d} s_{2} \mathrm{~d} s_{3}}{\sum_{i=1}^{3} \cos \frac{\Lambda_{1}^{i}}{2}\left(1+\cos \left(\frac{\Lambda_{1}^{i}}{2}-s_{i}\right)\right)}$.

For $U<0$, and $U<-\frac{2 B}{m}$, below of the continuous spectrum of the operator $\tilde{H}_{2 \Lambda_{1}}^{4}$ the function $D_{\Lambda_{1}}^{3}(z)$ has a single zero at the point $z_{1}<m_{\Lambda_{1}}^{3}$. For $U<0$, and $-\frac{2 B}{m} \leq U<0$, below of the continuous spectrum of the operator $\tilde{H}_{2 \Lambda_{1}}^{4}$ the function $D_{\Lambda_{1}}^{3}(z)$ cannot vanish.

$$
\text { Denote } M=\int_{T^{3}} \frac{\mathrm{d} s_{1} \mathrm{~d} s_{2} \mathrm{~d} s_{3}}{\sum_{i=1}^{3} \cos \frac{\Lambda_{1}^{i}}{2}\left(1-\cos \left(\frac{\Lambda_{1}^{i}}{2}-s_{i}\right)\right)} \text {. }
$$

For $U>0$ and $U>\frac{2 B}{M}$, above the continuous spectrum of the operator $\tilde{H}_{2 \Lambda_{1}}^{4}$ the function $D_{\Lambda_{1}}^{3}(z)$ has a single zero at the point $\tilde{z}_{1}>M_{\Lambda_{1}}^{3}$. For $0<U \leq \frac{2 B}{M}$, above the continuous spectrum of the operator $\tilde{H}_{2 \Lambda_{1}}^{4}$ the function $D_{\Lambda_{1}}^{3}(z)$ cannot vanish.

We now investigate the spectrum of the operator $\tilde{H}_{2 \Lambda_{2}}^{5}$ :

$$
\begin{aligned}
& \left(\tilde{H}_{2 \Lambda_{2}}^{5} f_{\Lambda_{2}}\right)(\gamma) \\
& =\left\{2 A+4 B \sum_{i=1}^{v} \cos \frac{\Lambda_{2}^{i}}{2} \cos \left(\frac{\Lambda_{2}^{i}}{2}-\gamma_{i}\right)\right\} f_{\Lambda_{2}}(\gamma)-2 U \int_{T^{v}} f_{\Lambda_{2}}(s) \mathrm{d} s .
\end{aligned}
$$


We set $D_{\Lambda_{2}}^{v}(z)=1-2 U \int_{T^{v}} \frac{\mathrm{d} s_{1} \mathrm{~d} s_{2} \cdots \mathrm{d} s_{v}}{2 A+4 B \sum_{i=1}^{v} \cos \frac{\Lambda_{2}^{i}}{2} \cos \left(\frac{\Lambda_{2}^{i}}{2}-s_{i}\right)-z}$.

The analogue of the Lemma 2 holds for the in this case. We consider the one-dimensional case.

Theorem 16. 1) At $v=1$ and $U<0$ and for all values of the parameter of the Hamiltonian, the operator $\tilde{H}_{2 \Lambda_{2}}^{5}$ has a unique eigenvalue $\tilde{z}_{2}=2 A+2 \sqrt{U^{2}+4 B^{2} \cos ^{2} \frac{\Lambda_{2}}{2}}$ that is above the continuous spectrum of $\tilde{H}_{2 \Lambda_{2}}^{5}$, i.e., $\tilde{z}_{2}>M_{\Lambda_{2}}^{1}$.

2) At $v=1$ and $U>0$ and for all values of the parameter of the Hamiltonian, the operator $\tilde{H}_{2 \Lambda_{2}}^{5}$ has a unique eigenvalue $z_{2}=2 A-2 \sqrt{U^{2}+4 B^{2} \cos ^{2} \frac{\Lambda_{2}}{2}}$ that is below the continuous spectrum of $\tilde{H}_{2 \Lambda_{2}}^{5}$, i.e., $z_{2}<m_{\Lambda_{2}}^{1}$.

Proof. If $U<0$, then in the one-dimensional case, the function $D_{\Lambda_{2}}^{v}(z)$ increases monotonically outside the continuous spectrum domain of the operator $\tilde{H}_{2 \Lambda_{2}}^{5}$. For $z<m_{\Lambda_{2}}^{1}$ the function $D_{\Lambda_{2}}^{v}(z)$ increases from 1 to $+\infty$, $D_{\Lambda_{2}}^{v}(z) \rightarrow 1$ as $z \rightarrow-\infty, D_{\Lambda_{2}}^{v}(z) \rightarrow+\infty$ as $z \rightarrow m_{\Lambda_{1}}^{1}-0$. Therefore, below the value $m_{\Lambda_{2}}^{1}$, the function $D_{\Lambda_{2}}^{v}(z)$ cannot vanish.

For $z>M_{\Lambda_{2}}^{1}$, and $U<0$, the function $D_{\Lambda_{2}}^{v}(z)$ increases from $-\infty$ to 1 , $D_{\Lambda_{2}}^{v}(z) \rightarrow-\infty$ as $z \rightarrow M_{\Lambda_{2}}^{1}+0, D_{\Lambda_{2}}^{v}(z) \rightarrow 1$ as $z \rightarrow+\infty$. Therefore, above the value $M_{\Lambda_{2}}^{1}$, the function $D_{\Lambda_{2}}^{v}(z)$ has a single zero at the point $z=\tilde{z}_{2}=2 A+2 \sqrt{U^{2}+4 B^{2} \cos ^{2} \frac{\Lambda_{2}}{2}}$. If $U>0$, then the function $D_{\Lambda_{2}}^{v}(z)$ decreases monotonically outside the continuous spectrum domain of the operator $\tilde{H}_{2 \Lambda_{2}}^{5}$. For $z<m_{\Lambda_{2}}^{1}$ the function $D_{\Lambda_{2}}^{v}(z)$ decreases from 1 to $-\infty$, $D_{\Lambda_{2}}^{v}(z) \rightarrow 1$ as $z \rightarrow-\infty, D_{\Lambda_{2}}^{v}(z) \rightarrow-\infty$ as $z \rightarrow m_{\Lambda_{2}}^{1}-0$. Therefore, below the value $m_{\Lambda_{2}}^{1}$, the function $D_{\Lambda_{2}}^{v}(z)$ has a single zero at the point $z=z_{2}=2 A-2 \sqrt{U^{2}+4 B^{2} \cos ^{2} \frac{\Lambda_{2}}{2}}$. For $z>M_{\Lambda_{2}}^{1}$, and $U>0$, the function $D_{\Lambda_{2}}^{v}(z)$ decreases from $+\infty$ to $1, D_{\Lambda_{2}}^{v}(z) \rightarrow+\infty \quad$ as $\quad z \rightarrow M_{\Lambda_{2}}^{1}+0$, $D_{\Lambda_{2}}^{v}(z) \rightarrow 1$ as $z \rightarrow+\infty$. Therefore, above the value $M_{\Lambda_{2}}^{1}$, the function $D_{\Lambda_{2}}^{v}(z)$ cannot vanish.

In the two-dimensional case, we have analogous results.

We consider three-dimensional case. Denote

$$
m=\int_{T^{3}} \frac{\mathrm{d} s_{1} \mathrm{~d} s_{2} \mathrm{~d} s_{3}}{\sum_{i=1}^{3} \cos \frac{\Lambda_{2}^{i}}{2}\left(1+\cos \left(\frac{\Lambda_{2}^{i}}{2}-s_{i}\right)\right)} .
$$

For $U>0$, and $U>\frac{2 B}{m}$, below of the continuous spectrum of the operator $\tilde{H}_{2 \Lambda_{2}}^{5}$ the function $D_{\Lambda_{2}}^{3}(z)$ has a single zero at the point $z_{2}<m_{\Lambda_{2}}^{3}$. For $U>0$, and $0<U \leq \frac{2 B}{m}$, below of the continuous spectrum of the operator 
$\tilde{H}_{2 \Lambda_{2}}^{5}$ the function $D_{\Lambda_{2}}^{3}(z)=0$ cannot vanish.

Denote $M=\int_{T^{3}} \frac{\mathrm{d} s_{1} \mathrm{~d} s_{2} \mathrm{~d} s_{3}}{\sum_{i=1}^{3} \cos \frac{\Lambda_{2}^{i}}{2}\left(1-\cos \left(\frac{\Lambda_{2}^{i}}{2}-s_{i}\right)\right)}$.

For $U<0$ and $U<-\frac{2 B}{M}$, above the continuous spectrum of the operator $\tilde{H}_{2 \Lambda_{2}}^{5}$ the function $D_{\Lambda_{2}}^{3}(z)$ has a single zero at the point $\tilde{z}_{2}>M_{\Lambda_{2}}^{3}$. For $-\frac{2 B}{M} \leq U<0$, above the continuous spectrum of the operator $\tilde{H}_{2 \Lambda_{2}}^{5}$ the function $D_{\Lambda_{2}}^{3}(z)$ cannot vanish.

We now investigate the spectrum of the operator

$$
\left(\tilde{H}_{2 \Lambda_{3}}^{6} f_{\Lambda_{3}}\right)(\lambda)=\left\{A+2 B \sum_{i=1}^{v} \cos \left(\Lambda_{3}^{i}-\lambda_{i}\right)\right\} f_{\Lambda_{3}}(\lambda)-2 U \int_{T^{v}} f_{\Lambda_{3}}(s) \mathrm{d} s .
$$

It is known that the continuous spectrum of $\tilde{H}_{2 \Lambda_{3}}^{6}$ is independent of $U$ and coincides with the segment $\sigma_{\text {cont }}\left(\tilde{H}_{2 \Lambda_{3}}^{6}\right)=[A-2 B v, A+2 B v]$.

We set $D_{\Lambda_{3}}^{v}(z)=1-2 U \int_{T^{v}} \frac{\mathrm{d} s_{1} \mathrm{~d} s_{2} \cdots \mathrm{d} s_{v}}{A+2 B \sum_{i=1}^{v} \cos \left(\Lambda_{3}^{i}-s_{i}\right)-z}$.

The analogue of the Lemma 2 holds for the in this case. We consider the one-dimensional case.

It is clear that the at $U<0 \quad(U>0)$ exists only one solution of the equation $D_{\Lambda_{3}}^{v}(z)=0$, the above (the below) the continuous spectrum of the operator $\tilde{H}_{2 \Lambda_{3}}^{6}$.

Theorem 17. 1) At $v=1$ and $U<0$ and for all values of the parameter of the Hamiltonian, the operator $\tilde{H}_{2 \Lambda_{3}}^{6}$ has a unique eigenvalue $\tilde{z}_{3}=A+2 \sqrt{U^{2}+B^{2}}$, that is above the continuous spectrum of operator $\tilde{H}_{2 \Lambda_{3}}^{6}$, i.e., $\tilde{z}_{3}>M_{\Lambda_{3}}^{1}$.

2) At $v=1$ and $U>0$ and for all values of the parameter of the Hamiltonian, the operator $\tilde{H}_{2 \Lambda_{3}}^{6}$ has a unique eigenvalue $z=z_{3}=A-2 \sqrt{U^{2}+B^{2}}$, that is below the continuous spectrum of operator $\tilde{H}_{2 \Lambda_{3}}^{6}$, i.e., $z_{3}<m_{\Lambda_{3}}^{1}$.

In the two-dimensional case to occur the analogous situation. For $U<0$, the function $D_{\Lambda_{3}}^{2}(z)$ above the continuous spectrum of the operator $\tilde{H}_{2 \Lambda_{3}}^{6}$ has a single zero at the point $\tilde{z}_{3}>M_{\Lambda_{3}}^{2}$. For $U>0$, the function $D_{\Lambda_{3}}^{2}(z)$ below the continuous spectrum of the operator $\tilde{H}_{2 \Lambda_{3}}^{6}$ has a single zero at the point $z_{3}<m_{\Lambda_{3}}^{2}$.

We now consider three-dimensional case. For $U>0$, and $U>\frac{3 B}{{ }^{6} W}$, the function $D_{\Lambda_{3}}^{2}(z)$ below the continuous spectrum of the operator $\tilde{H}_{2 \Lambda_{3}}^{6}$ has a single zero at the point $z_{3}<m_{\Lambda_{3}}^{3}$. For $0<U \leq \frac{3 B}{W}$, the function $D_{\Lambda_{3}}^{2}(z)$ below the continuous spectrum of the operator $\tilde{H}_{2 \Lambda_{3}}^{6}$ cannot vanish. For $U<0$, and $U<-\frac{3 B}{W}$, the function $D_{\Lambda_{3}}^{2}(z)$ above the continuous spectrum of the operator $\tilde{H}_{2 \Lambda_{3}}^{6}$ has a single zero at the point $\tilde{z}_{3}>M_{\Lambda_{3}}^{3}$. For $-\frac{3 B}{W} \leq U<0$, the 
function $D_{\Lambda_{3}}^{2}(z)$ above the continuous spectrum of the operator $\tilde{H}_{2 \Lambda_{3}}^{6}$ cannot vanish.

Now, using the obtained results and representation (14), we describe the structure of the essential spectrum and the discrete spectrum of the operator ${ }^{4} \tilde{H}_{1 / 2}^{d}$ fourth five-electron doublet state:

Theorem 18. At $v=1$ and $U<0$ the essential spectrum of the system of fourth five-electron doublet state operator ${ }^{4} \tilde{H}_{1 / 2}^{d}$ is exactly the union of seven segments.

$$
\begin{aligned}
\sigma_{e s s}\left({ }^{4} \tilde{H}_{1 / 2}^{d}\right)= & {[a+c+e, b+d+f] \cup\left[a+e+\tilde{z}_{2}, b+f+\tilde{z}_{2}\right] } \\
& \bigcup\left[c+e+z_{1}, d+f+z_{1}\right] \cup\left[c+z_{1}+\tilde{z}_{3}, d+z_{1}+\tilde{z}_{3}\right] \\
& \bigcup\left[a+c+\tilde{z}_{3}, b+d+\tilde{z}_{3}\right] \cup\left[a+\tilde{z}_{2}+\tilde{z}_{3}, b+\tilde{z}_{2}+\tilde{z}_{3}\right] \\
& \bigcup\left[e+z_{1}+\tilde{z}_{2}, f+z_{1}+\tilde{z}_{2}\right] .
\end{aligned}
$$

The discrete spectrum of the operator ${ }^{4} \tilde{H}_{1 / 2}^{d}$ consists of no more than one point: $\sigma_{\text {disc }}\left({ }^{4} \tilde{H}_{1 / 2}^{d}\right)=\left\{z_{1}+\tilde{z}_{2}+\tilde{z}_{3}\right\}$, or $\sigma_{\text {disc }}\left({ }^{4} \tilde{H}_{1 / 2}^{d}\right)=\varnothing$.

Here and hereafter $a=2 A-4 B \cos \frac{\Lambda_{1}}{2}, b=2 A+4 B \cos \frac{\Lambda_{1}}{2}$, $c=2 A-4 B \cos \frac{\Lambda_{2}}{2}, d=2 A+4 B \cos \frac{\Lambda_{2}}{2}, e=A-2 B, f=A+2 B$, $z_{1}=2 A-2 \sqrt{U^{2}+4 B^{2} \cos ^{2} \frac{\Lambda_{1}}{2}}, \quad \tilde{z}_{2}=2 A+2 \sqrt{U^{2}+4 B^{2} \cos ^{2} \frac{\Lambda_{2}}{2}}$, $\tilde{z}_{3}=A+2 \sqrt{U^{2}+B^{2}}$.

Theorem 19. At $v=1$ and $U>0$ the essential spectrum of the system of fourth five-electron doublet state operator ${ }^{4} \tilde{H}_{1 / 2}^{d}$ is exactly the union of seven segments:

$$
\begin{aligned}
\sigma_{e s s}\left({ }^{4} \tilde{H}_{1 / 2}^{d}\right)= & {[a+c+e, b+d+f] \cup\left[a+e+z_{2}, b+f+z_{2}\right] } \\
& \bigcup\left[c+e+\tilde{z}_{1}, d+f+\tilde{z}_{1}\right] \cup\left[e+\tilde{z}_{1}+z_{2}, f+\tilde{z}_{1}+z_{2}\right] \\
& \bigcup\left[a+c+z_{3}, b+d+z_{3}\right] \cup\left[a+z_{2}+z_{3}, b+z_{2}+z_{3}\right] \\
& \bigcup\left[c+\tilde{z}_{1}+z_{3}, d+\tilde{z}_{1}+z_{2}\right] .
\end{aligned}
$$

The discrete spectrum of the operator ${ }^{4} \tilde{H}_{1 / 2}^{d}$ consists of no more than one point: $\sigma_{\text {disc }}\left({ }^{4} \tilde{H}_{1 / 2}^{d}\right)=\left\{\tilde{z}_{1}+z_{2}+z_{3}\right\}$, or $\sigma_{\text {disc }}\left({ }^{4} \tilde{H}_{1 / 2}^{d}\right)=\varnothing$.

Here $\tilde{z}_{1}=2 A+2 \sqrt{U^{2}+4 B^{2} \cos ^{2} \frac{\Lambda_{1}}{2}}, \quad z_{2}=2 A-2 \sqrt{U^{2}+4 B^{2} \cos ^{2} \frac{\Lambda_{2}}{2}}$, $z_{3}=A-2 \sqrt{U^{2}+B^{2}}$.

In the two-dimensional case to occur the analogous results.

We now consider the three-dimensional case. Let $v=3$.

Theorem 20. The following statements hold:

1) Let $U<0$, and $U<-\frac{2 B}{m}, m<\frac{2}{3} W$, or $U<0, U<-\frac{3 B}{W}, m>\frac{2}{3} W$ or $U<0, U<-\frac{2 B}{M}, M<\frac{2}{3} W$. Then the essential spectrum of the system fourth five-electron doublet state operator ${ }^{4} \tilde{H}_{1 / 2}^{d}$ is the union of seven segments. 


$$
\begin{aligned}
\sigma_{e s s}\left({ }^{4} \tilde{H}_{1 / 2}^{d}\right)= & {[a+c+e, b+d+f] \cup\left[a+c+\tilde{z}_{3}, b+d+\tilde{z}_{3}\right] } \\
& \bigcup\left[a+e+\tilde{z}_{2}, b+f+\tilde{z}_{2}\right] \cup\left[a+\tilde{z}_{2}+\tilde{z}_{3}, b+\tilde{z}_{2}+\tilde{z}_{3}\right] \\
& \bigcup\left[c+e+z_{1}, d+f+z_{1}\right] \cup\left[c+z_{1}+\tilde{z}_{3}, d+z_{1}+\tilde{z}_{3}\right] \\
& \bigcup\left[e+z_{1}+\tilde{z}_{2}, f+z_{1}+\tilde{z}_{2}\right] .
\end{aligned}
$$

The discrete spectrum of the operator ${ }^{4} \tilde{H}_{1 / 2}^{d}$ consists of no more than one point. $\sigma_{\text {disc }}\left({ }^{4} \tilde{H}_{1 / 2}^{d}\right)=\left\{z_{1}+\tilde{z}_{2}+\tilde{z}_{3}\right\}$, or $\sigma_{\text {disc }}\left({ }^{4} \tilde{H}_{1 / 2}^{d}\right)=\varnothing$.

Here, $a=2 A-4 B \sum_{i=1}^{3} \cos \frac{\Lambda_{1}^{i}}{2}, \quad b=2 A+4 B \sum_{i=1}^{3} \cos \frac{\Lambda_{1}^{i}}{2}$, $c=2 A-4 B \sum_{i=1}^{3} \cos \frac{\Lambda_{2}^{i}}{2}, d=2 A+4 B \sum_{i=1}^{3} \cos \frac{\Lambda_{2}^{i}}{2}, \quad e=A-6 B, f=A+6 B$, and $z_{1}$ is an eigenvalue of the operator $\tilde{H}_{2 \Lambda_{1}}^{4}$, and $\tilde{z}_{2}$ is an eigenvalue of the operator $\tilde{H}_{2 \Lambda_{2}}^{5}$, and $\tilde{z}_{3}$ is an eigenvalue of the operator $\tilde{H}_{\Lambda_{3}}^{6}$.

2) Let $U<0,-\frac{2 B}{m} \leq U<-\frac{2 B}{M}$, and $M<\frac{2}{3} W$, or $U<0$, $-\frac{2 B}{m} \leq U<-\frac{3 B}{W}$, and $m>\frac{2}{3} W$, or $U<0,-\frac{3 B}{W} \leq U<-\frac{2 B}{m}$, and $M>m$, or $U<0,-\frac{3 B}{W} \leq U<-\frac{2 B}{M}$, and $M<m$, or $U<0,-\frac{2 B}{M} \leq U<-\frac{2 B}{m}$, and $m<\frac{2}{3} W$, or $U<0,-\frac{2 B}{M} \leq U<-\frac{3 B}{W}$, and $m>\frac{2}{3} W$, Then the essential spectrum of the system fourth five-electron doublet state operator ${ }^{4} \tilde{H}_{1 / 2}^{d}$ is the union of four segments.

$$
\begin{aligned}
\sigma_{e s s}\left({ }^{4} \tilde{H}_{1 / 2}^{d}\right)= & {[a+c+e, b+d+f] \cup\left[a+c+\tilde{z}_{3}, b+d+\tilde{z}_{3}\right] } \\
& \bigcup\left[a+e+\tilde{z}_{2}, b+f+\tilde{z}_{2}\right] \cup\left[a+\tilde{z}_{2}+\tilde{z}_{3}, b+\tilde{z}_{2}+\tilde{z}_{3}\right],
\end{aligned}
$$

or

$$
\begin{aligned}
\sigma_{e s s}\left({ }^{4} \tilde{H}_{1 / 2}^{d}\right)= & {[a+c+e, b+d+f] \cup\left[a+e+\tilde{z}_{2}, b+f+\tilde{z}_{2}\right] } \\
& \bigcup\left[c+e+z_{1}, d+f+z_{1}\right] \cup\left[e+z_{1}+\tilde{z}_{2}, f+z_{1}+\tilde{z}_{2}\right],
\end{aligned}
$$

or

$$
\begin{aligned}
\sigma_{e s s}\left({ }^{4} \tilde{H}_{1 / 2}^{d}\right)= & {[a+c+e, b+d+f] \cup\left[a+c+\tilde{z}_{3}, b+d+\tilde{z}_{3}\right] } \\
& \bigcup\left[c+e+z_{1}, d+f+z_{1}\right] \cup\left[c+z_{1}+\tilde{z}_{3}, d+z_{1}+\tilde{z}_{3}\right] .
\end{aligned}
$$

The discrete spectrum of the operator ${ }^{4} \tilde{H}_{1 / 2}^{d}$ is empty: $\sigma_{\text {disc }}\left({ }^{4} \tilde{H}_{1 / 2}^{d}\right)=\varnothing$.

3) Let $U<0,-\frac{2 B}{M} \leq U<-\frac{3 B}{W}$, and $M>m$, or $U<0,-\frac{2 B}{m} \leq U<-\frac{2 B}{M}$, and $m>\frac{2}{3} W$, or $U<0,-\frac{2 B}{m} \leq U<-\frac{3 B}{W}$, and $m>M$. Then the essential spectrum of the system fourth five-electron doublet state operator ${ }^{4} \tilde{H}_{1 / 2}^{d}$ is the union of two segments.

$$
\sigma_{e s s}\left({ }^{4} \tilde{H}_{1 / 2}^{d}\right)=[a+c+e, b+d+f] \cup\left[a+c+\tilde{z}_{3}, b+d+\tilde{z}_{3}\right],
$$

or

$$
\sigma_{\text {ess }}\left({ }^{4} \tilde{H}_{1 / 2}^{d}\right)=[a+c+e, b+d+f] \bigcup\left[a+e+\tilde{z}_{2}, b+f+\tilde{z}_{2}\right]
$$


or

$$
\sigma_{\text {ess }}\left({ }^{4} \tilde{H}_{1 / 2}^{d}\right)=[a+c+e, b+d+f] \bigcup\left[c+e+z_{1}, d+f+z_{1}\right]
$$

The discrete spectrum of the operator ${ }^{4} \tilde{H}_{1 / 2}^{d}$ is empty: $\sigma_{\text {disc }}\left({ }^{4} \tilde{H}_{1 / 2}^{d}\right)=\varnothing$.

4) Let $U<0,-\frac{3 B}{W} \leq U<0$ and $M<\frac{2}{3} W$, or $U<0$, and $-\frac{2 B}{M} \leq U<0$, and $M>m, M>\frac{2}{3} W$, or $U<0,-\frac{2 B}{m} \leq U<0, \quad m>\frac{2}{3} W$, and $M<m$.

Then the essential spectrum of the system fourth five-electron doublet state operator ${ }^{4} \tilde{H}_{1 / 2}^{d}$ is consists of single segment: $\sigma_{\text {ess }}\left({ }^{4} \tilde{H}_{1 / 2}^{d}\right)=[a+c+e, b+d+f]$, and the discrete spectrum of the operator ${ }^{4} \tilde{H}_{1 / 2}^{d}$ is empty: $\sigma_{\text {disc }}\left({ }^{4} \tilde{H}_{1 / 2}^{d}\right)=\varnothing$.

Let $v=3$.

Theorem 21. The following statements hold:

1) Let $U>0$, and $U>\frac{2 B}{M}, M<\frac{2}{3} W, M<m$, or $U>0$, and $U>\frac{3 B}{W}$, $M>\frac{2}{3} W$ or $U>0$, and $U>\frac{2 B}{m}, m<\frac{2}{3} W, M<m$. Then the essential spectrum of the system fourth five-electron doublet state operator ${ }^{4} \tilde{H}_{1 / 2}^{d}$ is the union of seven segments.

$$
\begin{aligned}
\sigma_{e s s}\left({ }^{4} \tilde{H}_{1 / 2}^{d}\right)= & {[a+c+e, b+d+f] \cup\left[a+c+z_{3}, b+d+z_{3}\right] } \\
& \bigcup\left[a+e+z_{2}, b+f+z_{2}\right] \cup\left[a+z_{2}+z_{3}, b+z_{2}+z_{3}\right] \\
& \bigcup\left[c+e+\tilde{z}_{1}, d+f+\tilde{z}_{1}\right] \cup\left[c+\tilde{z}_{1}+z_{3}, d+\tilde{z}_{1}+z_{3}\right] \\
& \bigcup\left[e+\tilde{z}_{1}+z_{2}, f+\tilde{z}_{1}+z_{2}\right] .
\end{aligned}
$$

The discrete spectrum of the operator ${ }^{4} \tilde{H}_{1 / 2}^{q}$ consists of no more than one point: $\sigma_{\text {disc }}\left({ }^{4} \tilde{H}_{1 / 2}^{d}\right)=\left\{\tilde{z}_{1}+z_{2}+z_{3}\right\}$, or $\sigma_{\text {disc }}\left({ }^{4} \tilde{H}_{1 / 2}^{d}\right)=\varnothing$.

Here, $\tilde{z}_{1}$ is an eigenvalue of the operator $\tilde{H}_{2 \Lambda_{1}}^{4}$, and $z_{2}$ is an eigenvalue of the operator $\tilde{H}_{2 \Lambda_{2}}^{5}$, and $z_{3}$ is an eigenvalue of the operator $\tilde{H}_{\Lambda_{3}}^{6}$.

2) Let $U>0, \frac{2 B}{M}<U \leq \frac{2 B}{m}$, and $M<\frac{2}{3} W$, or $U>0, \frac{3 B}{W}<U \leq \frac{2 B}{M}$, and $M<\frac{2}{3} W, M<m$, or $U>0, \frac{2 B}{M}<U \leq \frac{3 B}{W}$, and $M>\frac{2}{3} W, M<m$. Then the essential spectrum of the system fourth five-electron doublet state operator ${ }^{4} \tilde{H}_{1 / 2}^{d}$ is the union of four segments.

$$
\begin{aligned}
\sigma_{e s s}\left({ }^{4} \tilde{H}_{1 / 2}^{d}\right)= & {[a+c+e, b+d+f] \cup\left[a+e+z_{2}, b+f+z_{2}\right] } \\
& \bigcup\left[a+c+z_{3}, b+d+z_{3}\right] \cup\left[a+z_{2}+z_{3}, b+z_{2}+z_{3}\right],
\end{aligned}
$$

or

$$
\begin{aligned}
\sigma_{\text {ess }}\left({ }^{4} \tilde{H}_{1 / 2}^{d}\right)= & {[a+c+e, b+d+f] \cup\left[a+c+z_{3}, b+d+z_{3}\right] } \\
& \bigcup\left[c+e+\tilde{z}_{1}, d+f+\tilde{z}_{1}\right] \cup\left[c+\tilde{z}_{1}+z_{3}, d+\tilde{z}_{1}+z_{3}\right],
\end{aligned}
$$

or

$$
\begin{aligned}
\sigma_{\text {ess }}\left({ }^{4} \tilde{H}_{1 / 2}^{d}\right)= & {[a+c+e, b+d+f] \cup\left[a+e+z_{2}, b+f+z_{2}\right] } \\
& \bigcup\left[c+e+\tilde{z}_{1}, d+f+\tilde{z}_{1}\right] \cup\left[e+\tilde{z}_{1}+z_{2}, f+\tilde{z}_{1}+z_{2}\right] .
\end{aligned}
$$


The discrete spectrum of the operator ${ }^{4} \tilde{H}_{1 / 2}^{d}$ is empty: $\quad \sigma_{\text {disc }}\left({ }^{4} \tilde{H}_{1 / 2}^{d}\right)=\varnothing$.

3) Let $U>0, \frac{3 B}{W}<U \leq \frac{2 B}{M}$, and $m<M, M<\frac{2}{3} W$, or $U>0$, $\frac{2 B}{m}<U \leq \frac{3 B}{W}$, and $M<m, M<\frac{2}{3} W$, or $U>0, \frac{2 B}{m}<U \leq \frac{2 B}{M}$, and $M<m, M>\frac{2}{3} W$, or $U>0, \frac{2 B}{M}<U \leq \frac{2 B}{m}$, and $M>m, m>\frac{2}{3} W$. Then the essential spectrum of the system fourth five-electron doublet state operator ${ }^{4} \tilde{H}_{1 / 2}^{d}$ is the union of two segments:

$$
\sigma_{e s s}\left({ }^{4} \tilde{H}_{1 / 2}^{d}\right)=[a+c+e, b+d+f] \cup\left[a+c+z_{3}, b+d+z_{3}\right],
$$

or

$$
\sigma_{e s s}\left({ }^{4} \tilde{H}_{1 / 2}^{d}\right)=[a+c+e, b+d+f] \cup\left[c+e+\tilde{z}_{1}, d+f+\tilde{z}_{1}\right]
$$

or

$$
\sigma_{\text {ess }}\left({ }^{4} \tilde{H}_{1 / 2}^{d}\right)=[a+c+e, b+d+f] \bigcup\left[a+e+z_{2}, b+f+z_{2}\right] .
$$

The discrete spectrum of the operator ${ }^{4} \tilde{H}_{1 / 2}^{d}$ is empty. $\sigma_{\text {disc }}\left({ }^{4} \tilde{H}_{1 / 2}^{d}\right)=\varnothing$.

4) Let $U>0,0<U \leq \frac{3 B}{W}$, and $M<\frac{2}{3} W$, or $U>0,0<U \leq \frac{2 B}{m}$, and $m>\frac{2}{3} W$, or $U>0,0<U \leq \frac{2 B}{M}$, and $M<m, m>\frac{2}{3} W$. Then the essential spectrum of the system fourth five-electron doublet state operator ${ }^{4} \tilde{H}_{1 / 2}^{d}$ is consists of single segment: $\sigma_{\text {ess }}\left({ }^{4} \tilde{H}_{1 / 2}^{d}\right)=[a+c+e, b+d+f]$, and the discrete spectrum of the operator ${ }^{4} \tilde{H}_{1 / 2}^{d}$ is empty: $\sigma_{\text {disc }}\left({ }^{4} \tilde{H}_{1 / 2}^{d}\right)=\varnothing$.

Let $v=3$ and $\Lambda_{1}=\left(\Lambda_{1}^{0}, \Lambda_{1}^{0}, \Lambda_{1}^{0}\right)$, and $\Lambda_{2}=\left(\Lambda_{2}^{0}, \Lambda_{2}^{0}, \Lambda_{2}^{0}\right)$.

It is known that the continuous spectrum of $\tilde{H}_{2 \Lambda_{1}}^{4}$ is independent of $U$ and coincides with the segment $\sigma_{\text {cont }}\left(\tilde{H}_{2 \Lambda_{1}}^{4}\right)=\left[2 A-12 B \cos \frac{\Lambda_{1}^{0}}{2}, 2 A+12 B \cos \frac{\Lambda_{1}^{0}}{2}\right]$.

Theorem 22. At $v=3$ and $U<0$ and the total quasimomentum $\Lambda_{1}$ of the system have the form $\Lambda_{1}=\left(\Lambda_{1}^{1}, \Lambda_{1}^{2}, \Lambda_{1}^{3}\right)=\left(\Lambda_{1}^{0}, \Lambda_{1}^{0}, \Lambda_{1}^{0}\right)$. Then the operator $\tilde{H}_{2 \Lambda_{1}}^{4}$ has a unique eigenvalue $z_{1}$ if $U<-\frac{6 B \cos \frac{\Lambda_{1}^{0}}{2}}{W}$, that is below the continuous spectrum of operator $\tilde{H}_{2 \Lambda_{1}}^{4}$. Otherwise, the operator $\tilde{H}_{2 \Lambda_{1}}^{4}$ has no eigenvalue, that is below the continuous spectrum of operator $\tilde{H}_{2 \Lambda_{1}}^{4}$.

Theorem 23. At $v=3$ and $U>0$ and the total quasimomentum $\Lambda_{1}$ of the system have the form $\Lambda_{1}=\left(\Lambda_{1}^{1}, \Lambda_{1}^{2}, \Lambda_{1}^{3}\right)=\left(\Lambda_{1}^{0}, \Lambda_{1}^{0}, \Lambda_{1}^{0}\right)$. Then the operator $\tilde{H}_{2 \Lambda_{1}}^{4}$ has a unique eigenvalue $\tilde{z}_{1}$ if $U>\frac{6 B \cos \frac{\Lambda_{1}^{0}}{2}}{W}$, that is above the continuous spectrum of operator $\tilde{H}_{2 \Lambda_{1}}^{4}$. Otherwise, the operator $\tilde{H}_{2 \Lambda_{1}}^{4}$ has no eigenvalue, that is above the continuous spectrum of operator $\tilde{H}_{2 \Lambda_{1}}^{4}$.

It is known that the continuous spectrum of $\tilde{H}_{2 \Lambda_{2}}^{5}$ is independent of $U$ and 
coincides with the segment $\sigma_{\text {cont }}\left(\tilde{H}_{2 \Lambda_{2}}^{5}\right)=\left[2 A-12 B \cos \frac{\Lambda_{2}^{0}}{2}, 2 A+12 B \cos \frac{\Lambda_{2}^{0}}{2}\right]$.

Theorem 24. At $v=3$ and $U<0$ and the total quasimomentum $\Lambda_{2}$ of the system have the form $\Lambda_{2}=\left(\Lambda_{2}^{1}, \Lambda_{2}^{2}, \Lambda_{2}^{3}\right)=\left(\Lambda_{2}^{0}, \Lambda_{2}^{0}, \Lambda_{2}^{0}\right)$. Then the operator $\tilde{H}_{2 \Lambda_{2}}^{5}$ has a unique eigenvalue $\tilde{z}_{2}$ if $U<-\frac{6 B \cos \frac{\Lambda_{2}^{0}}{2}}{W}$, that is above the continuous spectrum of operator $\tilde{H}_{2 \Lambda_{2}}^{5}$. Otherwise, the operator $\tilde{H}_{2 \Lambda_{2}}^{5}$ has no eigenvalue, that is above the continuous spectrum of operator $\tilde{H}_{2 \Lambda_{2}}^{5}$.

Theorem 25. At $v=3$ and $U>0$ and the total quasimomentum $\Lambda_{2}$ of the system have the form $\Lambda_{2}=\left(\Lambda_{2}^{1}, \Lambda_{2}^{2}, \Lambda_{2}^{3}\right)=\left(\Lambda_{2}^{0}, \Lambda_{2}^{0}, \Lambda_{2}^{0}\right)$. Then the operator $\tilde{H}_{2 \Lambda_{2}}^{5}$ has a unique eigenvalue $z_{2}$ if $U>\frac{6 B \cos \frac{\Lambda_{2}^{0}}{2}}{W}$, that is below the continuous spectrum of operator $\tilde{H}_{2 \Lambda_{2}}^{5}$. Otherwise, the operator $\tilde{H}_{2 \Lambda_{2}}^{5}$ has no eigenvalue, that is below the continuous spectrum of operator $\tilde{H}_{2 \Lambda_{2}}^{5}$.

Now, using the obtained results and representation (14), we describe the structure of the essential spectrum and the discrete spectrum of the system fourth five-electron doublet state operator ${ }^{4} \tilde{H}_{1 / 2}^{d}$ :

Let $v=3$ and $\Lambda_{1}=\left(\Lambda_{1}^{0}, \Lambda_{1}^{0}, \Lambda_{1}^{0}\right)$, and $\Lambda_{2}=\left(\Lambda_{2}^{0}, \Lambda_{2}^{0}, \Lambda_{2}^{0}\right)$.

Theorem 26. The following statements hold:

1) Let $U<0, U<-\frac{6 B \cos \frac{\Lambda_{1}^{0}}{2}}{W}, \cos \frac{\Lambda_{1}^{0}}{2}>\cos \frac{\Lambda_{2}^{0}}{2}$, and $\cos \frac{\Lambda_{1}^{0}}{2}>\frac{1}{2}$, or $U<0, \quad U<-\frac{6 B \cos \frac{\Lambda_{2}^{0}}{2}}{W}, \quad \cos \frac{\Lambda_{1}^{0}}{2}<\cos \frac{\Lambda_{2}^{0}}{2}$ and $\cos \frac{\Lambda_{2}^{0}}{2}>\frac{1}{2}$, or $U<0$, $U<-\frac{3 B}{W}$, and $\cos \frac{\Lambda_{1}^{0}}{2}<\frac{1}{2}$. Then the essential spectrum of the system fourth five-electron doublet state operator ${ }^{4} \tilde{H}_{1 / 2}^{d}$ is consists of the union of seven segments:

$$
\begin{aligned}
\sigma_{e s s}\left({ }^{4} \tilde{H}_{1 / 2}^{d}\right)= & {\left[a_{1}+c_{1}+e_{1}, b_{1}+d_{1}+f_{1}\right] \cup\left[a_{1}+e_{1}+\tilde{z}_{2}, b_{1}+f_{1}+\tilde{z}_{2}\right] } \\
& \cup\left[c_{1}+e_{1}+z_{1}, d_{1}+f_{1}+z_{1}\right] \cup\left[e_{1}+z_{1}+\tilde{z}_{3}, f_{1}+z_{1}+\tilde{z}_{3}\right] \\
& \cup\left[a_{1}+c_{1}+\tilde{z}_{3}, b_{1}+d_{1}+\tilde{z}_{3}\right] \cup\left[a_{1}+\tilde{z}_{2}+\tilde{z}_{3}, b_{1}+\tilde{z}_{2}+\tilde{z}_{3}\right] \\
& \cup\left[c_{1}+z_{1}+\tilde{z}_{3}, d_{1}+z_{1}+\tilde{z}_{3}\right]
\end{aligned}
$$

The discrete spectrum of the operator ${ }^{4} \tilde{H}_{1 / 2}^{d}$ consists of no more than one point. $\sigma_{\text {disc }}\left({ }^{4} \tilde{H}_{1 / 2}^{d}\right)=\left\{z_{1}+\tilde{z}_{2}+\tilde{z}_{3}\right\}$, or $\sigma_{\text {disc }}\left({ }^{4} \tilde{H}_{1 / 2}^{d}\right)=\varnothing$.

Here and hereafter $a_{1}=2 A-12 B \cos \frac{\Lambda_{1}^{0}}{2}, b_{1}=2 A+12 B \cos \frac{\Lambda_{1}^{0}}{2}$, $c_{1}=2 A-12 B \cos \frac{\Lambda_{2}^{0}}{2}, \quad d_{1}=2 A+12 B \cos \frac{\Lambda_{2}^{0}}{2}, e_{1}=A-6 B, f_{1}=A+6 B$, and $z_{1}$ is an eigenvalue of the operator $\tilde{H}_{2 \Lambda_{1}}^{4}$, and $\tilde{z}_{2}$ is an eigenvalue of the operator $\tilde{H}_{2 \Lambda_{2}}^{5}$, and $\tilde{z}_{3}$ is an eigenvalue of the operator $\tilde{H}_{2 \Lambda_{3}}^{6}$. 
2) Let $U<0,-\frac{6 B \cos \frac{\Lambda_{1}^{0}}{2}}{W} \leq U<-\frac{6 B \cos \frac{\Lambda_{2}^{0}}{2}}{W}, \cos \frac{\Lambda_{1}^{0}}{2}>\cos \frac{\Lambda_{2}^{0}}{2}$, and $\cos \frac{\Lambda_{2}^{0}}{2}>\frac{1}{2}$, or $-\frac{6 B \cos \frac{\Lambda_{2}^{0}}{2}}{W} \leq U<-\frac{6 B \cos \frac{\Lambda_{1}^{0}}{2}}{W}, \cos \frac{\Lambda_{1}^{0}}{2}<\cos \frac{\Lambda_{2}^{0}}{2}$, and $\cos \frac{\Lambda_{1}^{0}}{2}>\frac{1}{2}$, or $U<0,-\frac{6 B \cos \frac{\Lambda_{2}^{0}}{2}}{W} \leq U<-\frac{3 B}{W}, \cos \frac{\Lambda_{2}^{0}}{2}>\frac{1}{2}$, and $\cos \frac{\Lambda_{1}^{0}}{2}<\frac{1}{2}$, or $U<0,-\frac{3 B}{W} \leq U<-\frac{6 B \cos \frac{\Lambda_{1}^{0}}{2}}{W}, \cos \frac{\Lambda_{1}^{0}}{2}<\frac{1}{2}$, and $\cos \frac{\Lambda_{1}^{0}}{2}>\cos \frac{\Lambda_{2}^{0}}{2}$. Then the essential spectrum of the system fourth five-electron doublet state operator ${ }^{4} \tilde{H}_{1 / 2}^{d}$ is consists of the union of four segments.

$$
\begin{aligned}
\sigma_{\text {ess }}\left({ }^{4} \tilde{H}_{1 / 2}^{d}\right)= & {\left[a_{1}+c_{1}+e_{1}, b_{1}+d_{1}+f_{1}\right] \cup\left[a_{1}+e_{1}+\tilde{z}_{2}, b_{1}+f_{1}+\tilde{z}_{2}\right] } \\
& \bigcup\left[a_{1}+c_{1}+\tilde{z}_{3}, b_{1}+d_{1}+\tilde{z}_{3}\right] \cup\left[a_{1}+\tilde{z}_{2}+\tilde{z}_{3}, b_{1}+\tilde{z}_{2}+\tilde{z}_{3}\right]
\end{aligned}
$$

or

$$
\begin{aligned}
\sigma_{e s s}\left({ }^{4} \tilde{H}_{1 / 2}^{d}\right)= & {\left[a_{1}+c_{1}+e_{1}, b_{1}+d_{1}+f_{1}\right] \cup\left[c_{1}+e_{1}+z_{1}, d_{1}+f_{1}+z_{1}\right] } \\
& \bigcup\left[a_{1}+c_{1}+\tilde{z}_{3}, b_{1}+d_{1}+\tilde{z}_{3}\right] \cup\left[c_{1}+z_{1}+\tilde{z}_{3}, d_{1}+z_{1}+\tilde{z}_{3}\right],
\end{aligned}
$$

or

$$
\begin{aligned}
\sigma_{e s s}\left({ }^{4} \tilde{H}_{1 / 2}^{d}\right)= & {\left[a_{1}+c_{1}+e_{1}, b_{1}+d_{1}+f_{1}\right] \cup\left[c_{1}+e_{1}+z_{1}, d_{1}+f_{1}+z_{1}\right] } \\
& \bigcup\left[a_{1}+e_{1}+\tilde{z}_{2}, b_{1}+f_{1}+\tilde{z}_{2}\right] \cup\left[e_{1}+z_{1}+\tilde{z}_{2}, f_{1}+z_{1}+\tilde{z}_{2}\right]
\end{aligned} .
$$

The discrete spectrum of the operator ${ }^{4} \tilde{H}_{1 / 2}^{d}$ is empty: $\sigma_{\text {disc }}\left({ }^{2} \tilde{H}_{1 / 2}^{d}\right)=\varnothing$.

3) Let $U<0,-\frac{6 B \cos \frac{\Lambda_{1}^{0}}{2}}{W} \leq U<-\frac{3 B}{W}, \cos \frac{\Lambda_{1}^{0}}{2}<\cos \frac{\Lambda_{2}^{0}}{2}$, and $\cos \frac{\Lambda_{1}^{0}}{2}>\frac{1}{2}$, or $-\frac{6 B \cos \frac{\Lambda_{2}^{0}}{2}}{W} \leq U<-\frac{3 B}{W}, \cos \frac{\Lambda_{1}^{0}}{2}>\cos \frac{\Lambda_{2}^{0}}{2}$, and $\cos \frac{\Lambda_{2}^{0}}{2}>\frac{1}{2}$, or $U<0$, $-\frac{3 B}{W} \leq U<-\frac{6 B \cos \frac{\Lambda_{1}^{0}}{2}}{W}, \cos \frac{\Lambda_{1}^{0}}{2}<\frac{1}{2}$, and $\cos \frac{\Lambda_{1}^{0}}{2}<\cos \frac{\Lambda_{2}^{0}}{2}$, or $U<0$, $-\frac{6 B \cos \frac{\Lambda_{1}^{0}}{2}}{W} \leq U<-\frac{6 B \cos \frac{\Lambda_{2}^{0}}{2}}{W}, \cos \frac{\Lambda_{1}^{0}}{2}>\cos \frac{\Lambda_{2}^{0}}{2}$, and $\cos \frac{\Lambda_{1}^{0}}{2}<\frac{1}{2}$. Then the essential spectrum of the system fourth five-electron doublet state operator ${ }^{4} \tilde{H}_{1 / 2}^{d}$ is consists of the union of two segments.

$$
\sigma_{e s s}\left({ }^{4} \tilde{H}_{1 / 2}^{d}\right)=\left[a_{1}+c_{1}+e_{1}, b_{1}+d_{1}+f_{1}\right] \cup\left[a_{1}+c_{1}+\tilde{z}_{3}, b_{1}+d_{1}+\tilde{z}_{3}\right],
$$

or

$$
\sigma_{e s s}\left({ }^{4} \tilde{H}_{1 / 2}^{d}\right)=\left[a_{1}+c_{1}+e_{1}, b_{1}+d_{1}+f_{1}\right] \bigcup\left[c_{1}+e_{1}+z_{1}, d_{1}+f_{1}+z_{1}\right]
$$

or 


$$
\sigma_{e s s}\left({ }^{4} \tilde{H}_{1 / 2}^{d}\right)=\left[a_{1}+c_{1}+e_{1}, b_{1}+d_{1}+f_{1}\right] \cup\left[a_{1}+e_{1}+\tilde{z}_{2}, b_{1}+f_{1}+\tilde{z}_{2}\right],
$$

The discrete spectrum of the operator ${ }^{4} \tilde{H}_{1 / 2}^{d}$ is empty: $\sigma_{\text {disc }}\left({ }^{4} \tilde{H}_{1 / 2}^{d}\right)=\varnothing$.

4) Let $U<0,-\frac{3 B}{W} \leq U<0, \cos \frac{\Lambda_{1}^{0}}{2}>\cos \frac{\Lambda_{2}^{0}}{2}$, and $\cos \frac{\Lambda_{2}^{0}}{2}>\frac{1}{2}$, or $U<0$, $-\frac{3 B}{W} \leq U<0, \cos \frac{\Lambda_{1}^{0}}{2}<\cos \frac{\Lambda_{2}^{0}}{2}$, and $\cos \frac{\Lambda_{1}^{0}}{2}>\frac{1}{2}$, or $U<0$,

$-\frac{6 B \cos \frac{\Lambda_{1}^{0}}{2}}{W} \leq U<0, \quad \cos \frac{\Lambda_{1}^{0}}{2}<\cos \frac{\Lambda_{2}^{0}}{2}$ and $\cos \frac{\Lambda_{1}^{0}}{2}<\frac{1}{2}$, or

$-\frac{6 B \cos \frac{\Lambda_{2}^{0}}{2}}{W} \leq U<0, \cos \frac{\Lambda_{1}^{0}}{2}>\cos \frac{\Lambda_{2}^{0}}{2}$ and $\cos \frac{\Lambda_{2}^{0}}{2}<\frac{1}{2}$. Then the essential spectrum of the system fourth five-electron doublet state operator ${ }^{4} \tilde{H}_{1 / 2}^{d}$ is consists of single segments: $\sigma_{\text {ess }}\left({ }^{4} \tilde{H}_{1 / 2}^{d}\right)=\left[a_{1}+c_{1}+e_{1}, b_{1}+d_{1}+f_{1}\right]$, and discrete spectrum of the operator ${ }^{4} \tilde{H}_{1 / 2}^{d}$ is empty: $\sigma_{\text {disc }}\left({ }^{4} \tilde{H}_{1 / 2}^{d}\right)=\varnothing$.

Theorem 27. The following statements hold:

1) Let $U>0, U>\frac{3 B}{W}, \cos \frac{\Lambda_{1}^{0}}{2}<\cos \frac{\Lambda_{2}^{0}}{2}$, and $\cos \frac{\Lambda_{2}^{0}}{2}<\frac{1}{2}$, or $U>0$, $U>\frac{3 B}{W}, \cos \frac{\Lambda_{1}^{0}}{2}>\cos \frac{\Lambda_{2}^{0}}{2}$, and $\cos \frac{\Lambda_{1}^{0}}{2}<\frac{1}{2}$, or $U>0, U>\frac{6 B \cos \frac{\Lambda_{1}^{0}}{2}}{W}$, $\cos \frac{\Lambda_{1}^{0}}{2}>\cos \frac{\Lambda_{2}^{0}}{2}$, and $\cos \frac{\Lambda_{1}^{0}}{2}>\frac{1}{2}$, or $U>0, U>\frac{6 B \cos \frac{\Lambda_{2}^{0}}{2}}{W}$, $\cos \frac{\Lambda_{1}^{0}}{2}<\cos \frac{\Lambda_{2}^{0}}{2}$, and $\cos \frac{\Lambda_{2}^{0}}{2}>\frac{1}{2}$. Then the essential spectrum of the system fourth five-electron doublet state operator ${ }^{4} \tilde{H}_{1 / 2}^{d}$ is consists of the union of seven segments.

$$
\begin{aligned}
\sigma_{e s s}\left({ }^{4} \tilde{H}_{1 / 2}^{d}\right)= & {\left[a_{1}+c_{1}+e_{1}, b_{1}+d_{1}+f_{1}\right] \cup\left[a_{1}+e_{1}+z_{2}, b_{1}+f_{1}+z_{2}\right] } \\
& \bigcup\left[c_{1}+e_{1}+\tilde{z}_{1}, d_{1}+f_{1}+\tilde{z}_{1}\right] \cup\left[e_{1}+\tilde{z}_{1}+z_{2}, f_{1}+\tilde{z}_{1}+z_{2}\right] \\
& \bigcup\left[a_{1}+c_{1}+z_{3}, b_{1}+d_{1}+z_{3}\right] \cup\left[a_{1}+z_{2}+z_{3}, b_{1}+z_{2}+z_{3}\right] \\
& \bigcup\left[c_{1}+\tilde{z}_{1}+z_{3}, d_{1}+\tilde{z}_{1}+z_{3}\right] .
\end{aligned}
$$

The discrete spectrum of the operator ${ }^{4} \tilde{H}_{1 / 2}^{d}$ consists of no more than one point. $\sigma_{\text {disc }}\left({ }^{4} \tilde{H}_{1 / 2}^{d}\right)=\left\{\tilde{z}_{1}+z_{2}+z_{3}\right\}$, or $\sigma_{\text {disc }}\left({ }^{4} \tilde{H}_{1 / 2}^{d}\right)=\varnothing$.

Here and hereafter, $\tilde{z}_{1}$ is an eigenvalue of the operator $\tilde{H}_{2 \Lambda_{1}}^{4}$, and $z_{2}$ is an eigenvalue of the operator $\tilde{H}_{2 \Lambda_{2}}^{5}$, and $z_{3}$ is an eigenvalue of the operator $\tilde{H}_{\Lambda_{3}}^{6}$.

2) Let $U>0, \frac{6 B \cos \frac{\Lambda_{2}^{0}}{2}}{W} \leq U<\frac{3 B}{W}, \cos \frac{\Lambda_{1}^{0}}{2}<\cos \frac{\Lambda_{2}^{0}}{2}$, and $\cos \frac{\Lambda_{2}^{0}}{2}<\frac{1}{2}$, or $U>0, \frac{6 B \cos \frac{\Lambda_{1}^{0}}{2}}{W} \leq U<\frac{3 B}{W}, \cos \frac{\Lambda_{1}^{0}}{2}>\cos \frac{\Lambda_{2}^{0}}{2}$, and $\cos \frac{\Lambda_{1}^{0}}{2}<\frac{1}{2}$, or $U>0$, 
$\frac{6 B \cos \frac{\Lambda_{1}^{0}}{2}}{W} \leq U<\frac{6 B \cos \frac{\Lambda_{2}^{0}}{2}}{W}, \cos \frac{\Lambda_{1}^{0}}{2}<\cos \frac{\Lambda_{2}^{0}}{2}$, and $\cos \frac{\Lambda_{1}^{0}}{2}>\frac{1}{2}$, or

$\frac{6 B \cos \frac{\Lambda_{2}^{0}}{2}}{W} \leq U<\frac{6 B \cos \frac{\Lambda_{1}^{0}}{2}}{W}, \cos \frac{\Lambda_{1}^{0}}{2}>\cos \frac{\Lambda_{2}^{0}}{2}$, and $\cos \frac{\Lambda_{2}^{0}}{2}>\frac{1}{2}$. Then the essential spectrum of the system fourth five-electron doublet state operator ${ }^{4} \tilde{H}_{1 / 2}^{d}$ is consists of the union of four segments.

$$
\begin{aligned}
\sigma_{e s s}\left({ }^{4} \tilde{H}_{1 / 2}^{d}\right)= & {\left[a_{1}+c_{1}+e_{1}, b_{1}+d_{1}+f_{1}\right] \cup\left[a_{1}+e_{1}+z_{2}, b_{1}+f_{1}+z_{2}\right] } \\
& \bigcup\left[c_{1}+e_{1}+\tilde{z}_{1}, d_{1}+f_{1}+\tilde{z}_{1}\right] \cup\left[e_{1}+\tilde{z}_{1}+z_{2}, f_{1}+\tilde{z}_{1}+z_{2}\right]
\end{aligned},
$$

or

$$
\begin{aligned}
\sigma_{e s s}\left({ }^{4} \tilde{H}_{1 / 2}^{d}\right)= & {\left[a_{1}+c_{1}+e_{1}, b_{1}+d_{1}+f_{1}\right] \cup\left[c_{1}+e_{1}+\tilde{z}_{1}, d_{1}+f_{1}+\tilde{z}_{1}\right] } \\
& \bigcup\left[a_{1}+c_{1}+z_{3}, b_{1}+d_{1}+z_{3}\right] \cup\left[c_{1}+\tilde{z}_{1}+z_{3}, d_{1}+\tilde{z}_{1}+z_{3}\right]
\end{aligned},
$$

or

$$
\begin{aligned}
\sigma_{e s s}\left({ }^{4} \tilde{H}_{1 / 2}^{d}\right)= & {\left[a_{1}+c_{1}+e_{1}, b_{1}+d_{1}+f_{1}\right] \cup\left[a_{1}+c_{1}+z_{3}, b_{1}+d_{1}+z_{3}\right] . } \\
& \bigcup\left[a_{1}+e_{1}+z_{2}, b_{1}+f_{1}+z_{2}\right] \cup\left[a_{1}+z_{2}+z_{3}, b_{1}+z_{2}+z_{3}\right]
\end{aligned} .
$$

The discrete spectrum of the operator ${ }^{4} \tilde{H}_{1 / 2}^{d}$ is empty: $\sigma_{\text {disc }}\left({ }^{4} \tilde{H}_{1 / 2}^{d}\right)=\varnothing$.

3) Let $U>0, \frac{6 B \cos \frac{\Lambda_{1}^{0}}{2}}{W} \leq U<\frac{6 B \cos \frac{\Lambda_{2}^{0}}{2}}{W}, \cos \frac{\Lambda_{1}^{0}}{2}<\cos \frac{\Lambda_{2}^{0}}{2}$, and $\cos \frac{\Lambda_{2}^{0}}{2}<\frac{1}{2}$, or $U>0, \frac{6 B \cos \frac{\Lambda_{2}^{0}}{2}}{W} \leq U<\frac{6 B \cos \frac{\Lambda_{1}^{0}}{2}}{W}, \cos \frac{\Lambda_{1}^{0}}{2}>\cos \frac{\Lambda_{2}^{0}}{2}$, and $\cos \frac{\Lambda_{1}^{0}}{2}<\frac{1}{2}$, or $U>0, \frac{3 B}{W} \leq U<\frac{6 B \cos \frac{\Lambda_{1}^{0}}{2}}{W}, \cos \frac{\Lambda_{1}^{0}}{2}<\cos \frac{\Lambda_{2}^{0}}{2}$, and $\cos \frac{\Lambda_{1}^{0}}{2}>\frac{1}{2}$, or $U>0, \frac{3 B}{W} \leq U<\frac{6 B \cos \frac{\Lambda_{2}^{0}}{2}}{W}, \cos \frac{\Lambda_{1}^{0}}{2}>\cos \frac{\Lambda_{2}^{0}}{2}$, and $\cos \frac{\Lambda_{2}^{0}}{2}>\frac{1}{2}$, or $U>0, \frac{6 B \cos \frac{\Lambda_{1}^{0}}{2}}{W} \leq U<\frac{3 B}{W}, \cos \frac{\Lambda_{1}^{0}}{2}<\cos \frac{\Lambda_{2}^{0}}{2}$, and $\cos \frac{\Lambda_{2}^{0}}{2}>\frac{1}{2}$, or $\frac{6 B \cos \frac{\Lambda_{2}^{0}}{2}}{W} \leq U<\frac{3 B}{W}, \cos \frac{\Lambda_{1}^{0}}{2}>\cos \frac{\Lambda_{2}^{0}}{2}$, and $\cos \frac{\Lambda_{1}^{0}}{2}>\frac{1}{2}$.

Then the essential spectrum of the system fourth five-electron doublet state operator ${ }^{4} \tilde{H}_{1 / 2}^{d}$ is consists of the union of two segments.

$$
\sigma_{e s s}\left({ }^{2} \tilde{H}_{1 / 2}^{d}\right)=\left[a_{1}+c_{1}+e_{1}, b_{1}+d_{1}+f_{1}\right] \cup\left[c_{1}+e_{1}+\tilde{z}_{1}, d_{1}+f_{1}+\tilde{z}_{1}\right],
$$

or

$$
\sigma_{\text {ess }}\left({ }^{4} \tilde{H}_{1 / 2}^{d}\right)=\left[a_{1}+c_{1}+e_{1}, b_{1}+d_{1}+f_{1}\right] \bigcup\left[a_{1}+e_{1}+z_{2}, b_{1}+f_{1}+z_{2}\right],
$$

or 


$$
\sigma_{e s s}\left({ }^{4} \tilde{H}_{1 / 2}^{d}\right)=\left[a_{1}+c_{1}+e_{1}, b_{1}+d_{1}+f_{1}\right] \cup\left[a_{1}+c_{1}+z_{3}, b_{1}+d_{1}+z_{3}\right] .
$$

The discrete spectrum of the operator ${ }^{4} \tilde{H}_{1 / 2}^{d}$ is empty. $\sigma_{\text {disc }}\left({ }^{4} \tilde{H}_{1 / 2}^{d}\right)=\varnothing$. 4) Let $U>0, \quad 0<U \leq \frac{3 B}{W}, \quad \cos \frac{\Lambda_{1}^{0}}{2}<\cos \frac{\Lambda_{2}^{0}}{2},\left(\cos \frac{\Lambda_{1}^{0}}{2}>\cos \frac{\Lambda_{2}^{0}}{2}\right)$, and $\cos \frac{\Lambda_{1}^{0}}{2}>\frac{1}{2}$, or $U>0,0<U \leq \frac{6 B \cos \frac{\Lambda_{1}^{0}}{2}}{W}, \cos \frac{\Lambda_{1}^{0}}{2}<\cos \frac{\Lambda_{2}^{0}}{2}$ and $\cos \frac{\Lambda_{2}^{0}}{2}<\frac{1}{2}$, or $0<U \leq \frac{6 B \cos \frac{\Lambda_{2}^{0}}{2}}{W}, \cos \frac{\Lambda_{1}^{0}}{2}>\cos \frac{\Lambda_{2}^{0}}{2}$, and $\cos \frac{\Lambda_{1}^{0}}{2}<\frac{1}{2}$. Then the essential spectrum of the system fourth five-electron doublet state operator ${ }^{4} \tilde{H}_{1 / 2}^{d}$ is consists of single segments: $\sigma_{\text {ess }}\left({ }^{4} \tilde{H}_{1 / 2}^{d}\right)=\left[a_{1}+c_{1}+e_{1}, b_{1}+d_{1}+f_{1}\right]$, and discrete spectrum of the operator ${ }^{4} \tilde{H}_{1 / 2}^{d}$ is empty. $\quad \sigma_{\text {disc }}\left({ }^{4} \tilde{H}_{1 / 2}^{d}\right)=\varnothing$.

\section{Conclusions}

In this paper, we consider the energy operator of five electron systems in the Hubbard model and describe the structure of the essential spectra and discrete spectrum of the system in the third and fourth doublet states. The obtained results show that in the one-dimensional and two-dimensional case, the essential spectrum of the system in the third doublet state is exactly the union of four segments, and the discrete spectrum is empty. In the three-dimensional case, the essential spectrum of the system in the third doublet state consists of the union of four segments, or the union of two segments, or consists of a single segment. The discrete spectrum of the system in the third doublet state always is empty. Consequently, the essential spectrum of the system in the third doublet state consists of the union of no more than four segments, and the discrete spectrum is empty.

In the case of the fourth doublet state, the essential spectrum of the system in the one-dimensional and two-dimensional case consists of union of seven segments, and the discrete spectrum of the system consists of most one point. In the three-dimensional case, the essential spectrum of the system in the fourth doublet state consists of the union of seven segments, or the union of four segments, or the union of two segments, or consists of a single segment. The discrete spectrum of the system in the fourth doublet state consists of no more than one point. Consequently, the essential spectrum of the system in the fourth doublet state consists of the union of no more than seven segments, and the discrete spectrum consists of only one point, or is empty.

\section{Conflicts of Interest}

The author declares no conflicts of interest regarding the publication of this paper.

\section{References}

[1] Hubbard, J. (1963) Electron Correlations in Narrow Energy Band. Proceedings of 
the Royal Society A, 276, 238-257. https://doi.org/10.1098/rspa.1963.0204

[2] Gutzwiller, M.C. (1963) Effect of Correlation on the Ferromagnetism of Transition Metals. Physical Review Letters, 10, 159-162. https://doi.org/10.1103/PhysRevLett.10.159

[3] Kanamori, J. (1963) Electron Correlation and Ferromagnetism of Transition Metals. Progress of Theoretical Physics, 30, 275-289. https://doi.org/10.1143/PTP.30.275

[4] Anderson, P.W. (1961) Localized Magnetic States in Metals. Physical Review, 124, 41-53. https://doi.org/10.1103/PhysRev.124.41

[5] Shubin, S.P. and Wonsowsky, S.V. (1934) On the Electron Theory of Metals. Proceedings of the Royal Society A, 145, 159-180. https://doi.org/10.1098/rspa.1934.0089

[6] Karpenko, B.V., Dyakin, V.V. and Budrina, G.L. (1986) Two Electrons in the Hubbard Model. Physics of Metals and Metallography, 61, 702-706.

[7] Mattis, D. (1986) The Few-Body Problems on a Lattice. Reviews of Modern Physics, 58, 370-379. https://doi.org/10.1103/RevModPhys.58.361

[8] Lieb, E. (1989) Two Theorems on the Hubbard Model. Physical Review Letters, 62, 1201-1204. https://doi.org/10.1103/PhysRevLett.62.1201

[9] Tsvelick, A.M. and Wiegman, P.B. (1983) Exact Results in the Theory of Magnetic Alloys. Advances in Physics, 32, 453-713. https://doi.org/10.1080/00018738300101581

[10] Izyumov, Yu.A. and Skryabin, Yu.N. (1987) Statistical Mechanics of Magnetically Ordered Systems. Nauka, Moscow; Consultants Bureau, New York, 1988. (In Russian)

[11] Tashpulatov, S.M. (2014) Spectral Properties of Three-Electron Systems in the Hubbard Model. Theoretical and Mathematical Physics, 179, 712-728. https://doi.org/10.1007/s11232-014-0173-y

[12] Tashpulatov, S.M. (2016) Spectra of the Energy Operator of Four-Electron Systems in the Triplet State in the Hubbard Mode. Journal of Physics: Conference Series, 697, Article ID: 012025. https://doi.org/10.1088/1742-6596/697/1/012025

[13] Tashpulatov, S.M. (2017) The Structure of Essential Spectra and Discrete Spectrum of Four-Electron Systems in the Hubbard Model in a Singlet State. Lobachevskii Journal of Mathematics, 38, 530-541. https://doi.org/10.1134/S1995080217030246

[14] Reed, M. and Simon, B. (1978) Methods of Modern Mathematical Physics, Vol. 1, Functional Analysis. Academic Press, New York, 267 p.

[15] Reed, M. and Simon, B. (1982) Methods of Modern Mathematical Physics, Vol. 4, Analysis of Operators. Academic Press, New York, 267 p.

[16] Ichinose, T. (1978) Spectral Properties of Tensor Products of Linear Operators, I. Transactions of the American Mathematical Society, 235, 75-113. https://doi.org/10.1090/S0002-9947-1978-0472915-2

[17] Ichinose, T. (1978) Spectral Properties of Tensor Products of Linear Operators, II: The Approximate Point Spectrum and Kato Essential Spectrum. Transactions of the American Mathematical Society, 237, 223-254. https://doi.org/10.2307/1997620

[18] Ichinose, T. (1982) On the Spectral Properties of Tensor Products of Linear Operators in Banach Spaces. Spectral Theory Banach Center Publications, Vol. 8, PWN-Polish Scientific Publishers, Warsaw, 294-300. https://doi.org/10.4064/-8-1-295-300

[19] Neimark, M.A. (1959) Normed Rings. Nauka, Moscow, 1968. English Transl. Prev. ed. Noordhoff, Gronongen. (In Russian) 
[20] Val'kov, V.V., Ovchinnikov, S.G. and Petrakovskii, O.P. (1988) The Excitation Spectra of Two-Magnon Systems in Easy-Axis Quasidimensional Ferromagnets. Soviet Physics—Solid State, 30, 3044-3047. 